\title{
Variational Master Field for Large-N Interacting Matrix Models - Free Random Variables on Trial
}

\author{
M. Engelhardt ${ }^{1,2, *, \dagger}$ and S. Levit ${ }^{1}$ \\ ${ }^{1}$ Department of Condensed Matter Physics \\ Weizmann Institute of Science \\ Rehovot 76100, Israel \\ ${ }^{2}$ Institut für theoretische Physik III \\ Universität Erlangen \\ Staudtstr.7, 91058 Erlangen, Germany
}

()

\begin{abstract}
Matrices are said to behave as free non-commuting random variables if the action which governs their dynamics constrains only their eigenvalues, i.e. depends on traces of powers of individual matrices. The authors use recently developed mathematical techniques in combination with a standard variational principle to formulate a new variational approach for matrix models. Approximate variational solutions of interacting large- $\mathrm{N}$ matrix models are found using the free random matrices as the variational space. Several classes of classical and quantum mechanical matrix models with different types of interactions are considered and the variational solutions compared with exact Monte Carlo and analytical results. Impressive agreement is found in a majority of cases.
\end{abstract}

PACS: 02.10.Sp, 11.15.Pg, 11.80.Fv, 12.38.-t

Keywords: Matrix models, large-N limit, variational method

\section{INTRODUCTION}

The main motivation behind this work lay in developing approximate analytical tools for dealing with the long range physics of Quantum Chromodynamics. The large- $N_{c}$ approximation is an attractive candidate for this project. Initiated by 't Hooft, Ref. [1], it was immediately applied to solve large- $N_{c} 1+1$ dimensional QCD, Refs. [2], [3], [4], [5] and led to extremely fruitful phenomenological insights and models, Refs. [3], [6], [7]. The factorization property of correlators of gauge invariant operators suggested the idea of the master field - the gauge field configuration which dominates the path integral in the large- $N_{c}$ limit, cf. Refs. [3], [6], [8]. Knowledge of the master field should allow to calculate any gauge invariant observable as if it were a classical non-fluctuating object.

A concrete example of a master field was provided by the exact solution of a model which described the dynamics (classical as well as quantum mechanical) of a single hermitian $N \times N$ matrix, Ref. 99. It was found that in the large- $N$ limit the Boltzmann integral (in the classical case) and the ground state properties (in the quantum case) of this model were completely determined by an ensemble of matrices with a frozen distribution of eigenvalues given by a solution of a certain integral equation of the mean field type. The "angular" orientations of the matrices in this ensemble, i.e. the unitary matrices $W$ in the decomposition $M=W m W^{+}$with $m=\operatorname{diag}\left(m_{1}, m_{2}, \ldots, m_{N}\right)$ were completely free, i.e. distributed with equal probability according to the unbiased $U(N)$ group theoretical Haar measure. This work and, in its sequel, Ref. [10] gave rise to an intense interest in solutions of large- $N$ matrix models and made contact with the study of such models in other fields of physics, most notably nuclear physics, cf. Ref. [11] and, more recently, two-dimensional quantum gravity, cf. Ref. [12] and quantum chaos, cf. Ref. [13]. The general interest in large- $N$ matrix models has been behind a steady progress in understanding solutions of various versions and generalizations of such models as reported e.g. in Refs. 14], [15], [16], 117, [18].

A new direction in this activity opened up when the physics community became acquainted with recent advances in the mathematical literature, associated with the work of Voiculescu, Ref. [19], on non-commutative probability theory, Ref. [20]. The statistical mechanics (classical or quantum mechanical) of matrix (non-commuting) variables or (gauge) fields is an example of probability theory of non-commutative variables. A concept which turned out to be especially useful for the study of large- $N$ matrix and gauge theories, cf. Refs. [21] and [22], was the introduction in Ref. 19] of the so-called free random variables. The simplest examples of such variables occur in independent

\footnotetext{
*Address from 1.10.96: Institut für theoretische Physik, Universität Tübingen, Auf der Morgenstelle 14, 72076 Tübingen, Germany.

${ }^{\dagger}$ Supported in part by MINERVA and by the Bundesministerium für Bildung und Forschung, Germany.
} 
large- $N$ matrix models, i.e. in the classical statistical mechanics of several $N \times N, \quad N \rightarrow \infty$ hermitian matrices $M_{i}, i=1, \ldots, D$, with the probability distribution (the Boltzmann factor) given as $\operatorname{const} \cdot \exp \left(-N^{2} \sum_{i} V_{i}\left(M_{i}\right)\right)$ with $V_{i}\left(M_{i}\right)=(1 / N) \operatorname{Tr} P_{i}\left(M_{i}\right)$ and $P_{i}$ polynomial functions. Typical "observables" in such a model are correlators

$$
\frac{1}{N}\left\langle\operatorname{Tr}\left(M_{i_{1}} \ldots M_{i_{k}}\right)\right\rangle \equiv \int \frac{1}{N} \operatorname{Tr}\left(M_{i_{1}} \ldots M_{i_{k}}\right) \exp \left\{\sum_{i=1}^{D}\left[F_{i}-N^{2} V_{i}\left(M_{i}\right)\right]\right\} \prod_{k=1}^{D} D \mu\left[M_{k}\right],
$$

with the integration measure

$$
D \mu\left[M_{k}\right] \equiv \prod_{\gamma=1}^{N} d\left(M_{k}\right)_{\gamma \gamma} \prod_{\gamma>\nu=1}^{N} d \operatorname{Re}\left(M_{k}\right)_{\gamma \nu} d \operatorname{Im}\left(M_{k}\right)_{\gamma \nu},
$$

and $F_{i}=-\ln \int D \mu\left[M_{i}\right] \exp \left[-N^{2} V_{i}\left(M_{i}\right)\right]$. Were the $M_{i}$ regular commuting variables, one would call them independent since $\left\langle M_{i_{1}} \ldots M_{i_{k}}\right\rangle=\left\langle M_{i_{1}}\right\rangle \ldots\left\langle M_{i_{k}}\right\rangle$ for any selection $i_{1}, \ldots, i_{k}$. For the matrix model this is obviously not the case. The probability distribution depends only upon the eigenvalues $m_{i}$ of the $M_{i}$. The expectation of products of the $M_{i}$ on the other hand involves also the "angular" variables $W_{i}$ in the decomposition $M_{i}=W_{i} m_{i} W_{i}^{+}$. These variables however are "free", meaning that they are weighted with the unbiased Haar $U(N)$ measure. The integrals over the $W_{i}$ can therefore be evaluated in a universal manner independent of what the potentials $V_{i}$ are. The remaining integrals over the $m_{i}$ can then be evaluated in the limit $N \rightarrow \infty$ by a saddle point approximation as in Ref. [9]. The remarkable fact is that this procedure can be formulated in terms of very general rules relating any correlator of independent matrices to a linear combination of products of various individual moments of these matrices, cf. [19. These rules will be reviewed in Section II B below. Any non-commutative variables distributed in such a manner that they satisfy these rules are termed "free random variables". Refs. 221] and 22 provide a review of the techniques which were developed to deal with such things as sums and products of free random variables, to define a certain operator algebra in a suitably defined Fock space, and further applications, cf. also [23], 24], 25].

It has been possible to prove that a master field for any matrix model can be constructed on the basis of the operator algebra developed for free models, Ref. [21], 22]. However, so far no dynamical principle has been advanced in order to carry out this construction explicitely for interacting matrix and field theories. This paper presents an attempt to make progress in this direction by using a variational method based on the inequality $\left\langle e^{-S}\right\rangle \geq e^{-\langle S\rangle}$. This approach is fairly standard in statistical physics. The new element will be to use the free matrix models as the trial variational space. The details of the development are outlined in Section II below. Given a certain set of variables in terms of which the action is formulated, one can give a complete solution of the variational problem in the space of free matrix models by defining what will be called the free partner of the exact action of the model. Subsequently one observes however that the available variational freedom is even wider since one can first transform to a new set of variables, $M_{i} \rightarrow M_{i}\left(B_{j}\right)$. In the new $B_{i}$ it is again possible to define the free partner of the action and give the general variational solution. Thus the "art part" of the proposed variational method is the choice of the optimal $B_{i}$ or in other words of the optimal parameters of the transformation $M_{i} \rightarrow M_{i}\left(B_{j}\right)$.

Unfortunately it is hard to give estimates of the accuracy of this variational approximation. As usual, one must gain experience from examples and comparison with exact solutions. In Section III, a number of such examples are considered starting with classical interacting matrices and then going to quantum models of first one and then two interacting quantum matrices. Only linear transformations $M_{i}=\sum_{j} c_{i j} B_{j}$ are considered and the variational results using free random variables are compared with exact Monte Carlo calculations and, where available, with analytic results. In almost all models considered, e.g. in the models of two interacting matrices with the action

$$
S=\frac{1}{N} \operatorname{Tr}\left(M_{1}^{4}+M_{2}^{4}+g M_{1}^{2} M_{2}^{2}\right)
$$

and various values of $g$, of many interacting matrices with

$$
S=\frac{1}{D-1} \sum_{i \neq j}^{D} \frac{1}{N} \operatorname{Tr} M_{i}^{2} M_{j}^{2} \quad \text { and } \quad S=\frac{1}{D-1} \sum_{i \neq j}^{D} \frac{1}{N} \operatorname{Tr} M_{i}^{4} M_{j}^{4}
$$

and in the quantum models of one and two interacting matrices, impressively good agreement is found with exact results. Some models are also presented for which the method (with the additional limitation to the linear $M \rightarrow B$ transformations) failed completely. Presumably nonlinear transformations could improve the agreement but the authors have not investigated this. 


\section{VARIATIONAL METHOD FOR MATRIX MODELS}

In this paper, matrix models described by partition functions of the type

$$
Z \equiv e^{-F}=\int \exp \left(-N^{2} S\left[M_{1}, \ldots, M_{D}\right]\right) \prod_{k=1}^{D} D \mu\left[M_{k}\right]
$$

are considered, with $M_{k}, k=1, \ldots, D$, representing hermitian $N \times N$ dimensional matrices, in the limit $N \rightarrow \infty$. A factor $N^{2}$ has been pulled out in the exponent and in return, all matrix traces appearing in actions $S$ will be accompanied by a factor $1 / N$. Note that the term "action" is used very loosely here to denote essentially any weight appearing in the exponent of the Boltzmann factor in a partition function. Only actions $S\left[M_{1}, \ldots, M_{D}\right]$ which are invariant under the global unitary "rotations" $M_{i} \rightarrow U M_{i} U^{+}$will be considered. One can further distinguish between "classical" and "quantum" matrix models. By classical one means the models for which the above integral is just the ordinary integral with the integration measure given by (2) whereas in quantum models this is a path integral with $M_{i}$ depending on (imaginary) time $\tau, M_{i}(\tau)$, and the measure

$$
\mathcal{N} \prod_{0<\tau<\beta} D \mu\left[M_{k}(\tau)\right]=\mathcal{N} \prod_{0<\tau<\beta} \prod_{\gamma=1}^{N} d\left(M_{k}(\tau)\right)_{\gamma \gamma} \prod_{\gamma>\nu=1}^{N} d \operatorname{Re}\left(M_{k}(\tau)\right)_{\gamma \nu} d \operatorname{Im}\left(M_{k}(\tau)\right)_{\gamma \nu} .
$$

Here $\beta$ is the inverse temperature and $\mathcal{N}$ is a normalization factor which in principle depends on $\Delta \tau$, but will not be important in the following.

In both the classical and quantum cases one deals with integrals over many matrix variables and the real distinction will lie in the form of the action. In the classical case the action (the classical potential) is a real function usually represented as a sum of monomials in powers of the $M_{i}$,

$$
S_{\mathrm{cl}} \equiv V\left(M_{1}, \ldots, M_{D}\right)=\frac{1}{N} \sum_{i_{1}, i_{2}, \ldots, i_{D}} \sum_{m_{1}, m_{2}, \ldots, m_{D}} C_{m_{1} m_{2} \ldots m_{D}}^{i_{1}, i_{2}, \ldots, i_{D}} \operatorname{Tr}\left(M_{i_{1}}^{m_{1}} M_{i_{2}}^{m_{2}} \ldots M_{i_{D}}^{m_{D}}\right)
$$

with real coefficients $C_{m_{1} m_{2} \ldots m_{D}}^{i_{1}, i_{2}, \ldots, i_{D}}$. In the quantum case the action will be a sum of kinetic and potential terms

$$
S_{Q}=\frac{1}{2 \Delta \tau} \sum_{0<\tau<\beta} \sum_{i=1}^{D} \frac{1}{N} \operatorname{Tr}\left[M_{i}(\tau+\Delta \tau)-M_{i}(\tau)\right]^{2}+\sum_{0<\tau<\beta} \Delta \tau V\left(M_{1}(\tau), \ldots, M_{D}(\tau)\right),
$$

with $V$ as in (5).

\section{A. The Variational Principle}

As was reviewed in the Introduction, only a limited number of matrix models are amenable to analytical solution so that various methods of approximation are called for. Here, the powers of the variational method, combined with the large- $N$ saddle point evaluation, will be explored. As is common with statistical integrals, the variational approximation will be developed on the basis of the inequality, cf. Ref. [26],

$$
\left\langle e^{-x}\right\rangle \geq e^{-\langle x\rangle}
$$

which expresses the convexity property of the exponential function - the average value of the exponential of a random variable $x$ is greater than or equal to the exponential of the average provided $x$ is real and the weights used in the averaging are positive.

If one can find an action $S_{0}[M]$ which is simple enough to allow the calculation of integrals like

$$
\int \prod_{k} D \mu\left[M_{k}\right] e^{-N^{2} S_{0}[M]} \text { and } \int \prod_{k} D \mu\left[M_{k}\right] K\left(M_{1}, \ldots, M_{D}\right) e^{-N^{2} S_{0}[M]}
$$

for some simple functions $K$ then one can approximate the integral (3) as follows. One can represent

$$
e^{-F}=\int \prod_{k} D \mu\left[M_{k}\right] e^{-N^{2} S[M]}=\int \prod_{k} D \mu\left[M_{k}\right] e^{-N^{2}\left(S[M]-S_{0}[M]\right)} e^{-N^{2} S_{0}[M]}=e^{-F_{0}}\left\langle e^{-N^{2}\left(S-S_{0}\right)}\right\rangle_{S_{0}} .
$$


where $\langle O\rangle_{S}$ means $\int O \exp \left(F-N^{2} S\right) D \mu(M)$. Using the inequality (7) one obtains

$$
e^{-F}=e^{-F_{0}}\left\langle e^{-N^{2}\left(S-S_{0}\right)}\right\rangle_{S_{0}} \geq e^{-\left[F_{0}+N^{2}\left\langle S-S_{0}\right\rangle_{S_{0}}\right]},
$$

or

$$
F \leq F_{0}+N^{2}\left\langle S-S_{0}\right\rangle_{S_{0}}
$$

If $S_{0}$ depends on adjustable parameters, one can find their optimal values by minimizing the right hand side of the above inequality. The resulting $S_{0}$ can then be adopted as an approximation for $S$ with which one should calculate the relevant correlators. As always with variational methods, it is hard to assess the accuracy of the approximation. Here, it will be tested in different classes of matrix models, using the so-called free matrix models (cf. below) as the variational space, by comparing (where available) with exact analytical results or with Monte Carlo simulations.

\section{B. Free Matrix Models}

Freeness is a concept recently developed in the mathematical literature associated with noncommuting random variables [19]. While freeness is abstractly defined without reference to a specific realization of the random variables, in the context discussed here, namely in relation to large- $N$ hermitian multi-matrix models, it essentially means that the weight $\exp \left(F-N^{2} S\right)$ according to which the matrices are distributed contains no bias with respect to the relative $S U(N \rightarrow \infty)$ "orientations" of the matrices. In terms of the decomposition $M_{k}=W_{k} m_{k} W_{k}^{+}$with diagonal $m_{k}$ and unitary $W_{k}$ this means that the action $S$ in the free models depends only on the $m_{k}$. Consequently all the angular integrals in any given correlation function are weighted only with the group theoretical $U(N)$ Haar measure and give universal results independent of what the action is. The remaining integrals over the $m_{k}$ in the large- $N$ limit are controlled by the saddle point of the effective action which includes the contribution from the Vandermonde determinants for each $k$, cf. examples below. As a consequence of these properties all correlation functions for free multi-matrix models are systematically accessible in terms of the moments of individual matrices through the recently developed techniques, as will be elaborated upon below, cf. also Refs. [19], 21], [22].

A family of noncommuting random variables $M_{i}$ is called free if and only if

$$
\left\langle f_{1}\left(M_{i_{1}}\right) f_{2}\left(M_{i_{2}}\right) \ldots f_{n}\left(M_{i_{n}}\right)\right\rangle=0 \text { whenever }\left\langle f_{j}\left(M_{i_{j}}\right)\right\rangle=0 \text { for all } j
$$

where $i_{j} \neq i_{j+1}$ (and $i_{n+1}$ is identified with $i_{1}$ ). Note that the bracket notation introduced here following 19. means taking the expectation value of the trace of the expression inside, divided by $N$. In order to evaluate a general correlation function $\left\langle p_{1}\left(M_{i_{1}}\right) p_{2}\left(M_{i_{2}}\right) \ldots p_{n}\left(M_{i_{n}}\right)\right\rangle$, one can use

$$
\left\langle\left(p_{1}\left(M_{i_{1}}\right)-\left\langle p_{1}\left(M_{i_{1}}\right)\right\rangle\right)\left(p_{2}\left(M_{i_{2}}\right)-\left\langle p_{2}\left(M_{i_{2}}\right)\right\rangle\right) \ldots\left(p_{n}\left(M_{i_{n}}\right)-\left\langle p_{n}\left(M_{i_{n}}\right)\right\rangle\right)\right\rangle=0
$$

and multiply out the terms in the expectation value. This recursively determines correlation functions of a certain order in terms of lower-order correlation functions until one arrives at an expression containing only the moments of the individual matrices. For two free matrices $M_{1}$ and $M_{2}$ this algorithm yields, e.g.,

$$
\begin{aligned}
\left\langle p_{1}\left(M_{1}\right) p_{2}\left(M_{2}\right)\right\rangle & =\left\langle p_{1}\left(M_{1}\right)\right\rangle\left\langle p_{2}\left(M_{2}\right)\right\rangle \\
\left\langle M_{1} M_{2} M_{1} M_{2}\right\rangle & =\left\langle M_{1}^{2}\right\rangle\left\langle M_{2}\right\rangle^{2}+\left\langle M_{2}^{2}\right\rangle\left\langle M_{1}\right\rangle^{2}-\left\langle M_{1}\right\rangle^{2}\left\langle M_{2}\right\rangle^{2}
\end{aligned}
$$

Building on this information, it has been possible, e.g., to determine, [19], how the eigenvalue distributions of matrices which are free with respect to one another are additively and multiplicatively convoluted.

Freeness in matrix models is a slightly more general concept than what is usually meant by the term "independent matrix model", in which the potential governing the distribution of the matrices is a sum of terms for each individual matrix. Independent matrix models are certainly free; however, so is e.g. a two-matrix model with potential

$$
V\left(M_{1}, M_{2}\right)=\frac{1}{N} \operatorname{Tr} M_{1}^{2}+\frac{1}{N} \operatorname{Tr} M_{2}^{2}+\frac{1}{N^{2}} \operatorname{Tr} M_{1}^{2} \operatorname{Tr} M_{2}^{2} .
$$

More generally, the potential of a free matrix model can be any function of traces over functions of the individual matrices". Such more general free matrix models are almost as easy to solve as the independent ones. This is

\footnotetext{
${ }^{1}$ To the authors' knowledge, this type of model was first considered in 27].
} 
best illustrated by an example. Consider the model described by (15). After the transformation of variables $M_{i}=$ $W_{i} m_{i} W_{i}^{+}$, with diagonal $m_{i}$ and unitary $W_{i}$, the potential $V\left(M_{1}, M_{2}\right)$ depends only on the $m_{i}$. Moreover, the transformation introduces the Vandermonde Jacobians which, when exponentiated, modify the potential into

$$
\begin{aligned}
N^{2} V_{\text {eff }} & =\sum_{i=1,2} \sum_{\alpha \neq \beta}^{N} \ln \left|m_{i, \alpha}-m_{i, \beta}\right|+N^{2} V\left(m_{1}, m_{2}\right)= \\
& =N^{2}\left[\sum_{i=1,2}\left(\int_{-\infty}^{\infty} \rho_{i}(\mu) \ln \left|\mu-\mu^{\prime}\right| \rho_{i}\left(\mu^{\prime}\right) d \mu d \mu^{\prime}+\int_{-\infty}^{\infty} \rho_{i}(\mu) \mu^{2}\right)+\prod_{i=1,2} \int_{-\infty}^{\infty} \rho_{i}(\mu) \mu^{2} d \mu\right] .
\end{aligned}
$$

In the large- $N$ limit, the distributions $\rho_{i}(\mu)$ of the eigenvalues are "frozen" at a saddle point, so that the moments of the matrices have definite values

$$
\frac{1}{N} \operatorname{Tr} M_{1}^{2}=x_{1} \quad \frac{1}{N} \operatorname{Tr} M_{2}^{2}=x_{2}
$$

This means that the saddle point eigenvalue distributions $\rho_{i}(\mu)$ can be solved for as if they were controlled by the potentials

$$
V\left(M_{1}\right)=\left(x_{2}+1\right) \frac{1}{N} \operatorname{Tr} M_{1}^{2} \quad V\left(M_{2}\right)=\left(x_{1}+1\right) \frac{1}{N} \operatorname{Tr} M_{2}^{2} .
$$

For such quadratic potentials, one obtains the well-known semicircular distributions, cf. Ref. [11], [9]

$$
\rho_{1}(\mu)=\frac{x_{2}+1}{\pi} \sqrt{\frac{2}{x_{2}+1}-\mu^{2}} \quad \rho_{2}(\mu)=\frac{x_{1}+1}{\pi} \sqrt{\frac{2}{x_{1}+1}-\mu^{2}} .
$$

The constants $x_{1}$ and $x_{2}$ can now be determined by the self-consistency conditions

$$
\left.\begin{array}{l}
x_{1}=\left\langle M_{1}^{2}\right\rangle=\int d \mu \mu^{2} \rho_{1}(\mu) \\
x_{2}=\left\langle M_{2}^{2}\right\rangle=\int d \mu \mu^{2} \rho_{2}(\mu)
\end{array}\right\} \Rightarrow x_{1}=x_{2}=\frac{\sqrt{3}-1}{2} .
$$

Including such self-consistency conditions on selected moments of the matrices is the only additional step needed compared with solving independent matrix models. Knowledge of the eigenvalue distributions $\rho_{i}(\mu)$ is sufficient to calculate any correlation function since the latter can be reduced using Eq. (12) to a sum of products of individual moments, i.e. to the integrals $\left\langle M_{i}^{k}\right\rangle=\int \mu^{k} \rho_{i}(\mu) d \mu$.

It was argued e.g. in Refs. [21], [22 that a free behaviour of the matrix variables representing physical degrees of freedom may capture correctly the main features of the dynamics in the large- $N$ limit of nonabelian gauge theories. This hope and the wealth of available analytical techniques presented above render the space of free matrix models a good candidate for variational calculations. The main idea of the present approach is to choose the optimal action $S_{0}$ in the variational principle (10) from among all actions which leave the matrices in terms of which $S$ is given, free with respect to one another.

\section{General Variational Solution in the Space of Free Matrix Models}

In making a variational approximation based on free matrix models one must first decide which combinations of the original matrix variables $M_{i}$ will be assumed to be free, i.e. in which variables to formulate the exact action $S$. One should realize that e.g. free $M_{1}, M_{2}$ do not imply free $M_{1}+M_{2}, M_{1}-M_{2}$ nor vice versa (more about this below). The second step is to find the best trial action $S_{0}$ which is free in the variables one has settled for.

In this subsection, the solution of the second step is addressed. In other words, consider $S_{0}$ to be free in terms of the original $M_{i}$. Under this limitation, it is possible to give a general recipe for finding the trial $S_{0}$ which minimizes the right hand side of $(10)$. For this it is useful to introduce the following algorithm. Given a trace of any function of matrices $M_{i}, P=(1 / N) \operatorname{Tr} p\left(M_{1}, \ldots, M_{D}\right)$, write down its expectation value in terms of the moments of the individual matrices under the assumption that the matrices are free with respect to one another. This is easily accomplished for any multinomial expression using Eq. (12). Now rewrite the resulting expression with $\left\langle M_{i}^{j}\right\rangle$ replaced by $\operatorname{Tr} M_{i}^{j} / N$. This defines what will be called the "free partner" $P_{f}$ of $P$ (the "liberated" $P$, so to speak). For instance, the free partner of $P=(1 / N) \operatorname{Tr}\left(M_{1} M_{2} M_{1} M_{2}\right)$ is

$$
P_{f}=\frac{1}{N^{3}} \operatorname{Tr} M_{1}^{2}\left(\operatorname{Tr} M_{2}\right)^{2}+\frac{1}{N^{3}}\left(\operatorname{Tr} M_{1}\right)^{2} \operatorname{Tr} M_{2}^{2}-\frac{1}{N^{4}}\left(\operatorname{Tr} M_{1}\right)^{2}\left(\operatorname{Tr} M_{2}\right)^{2},
$$


cf. Eq. (14). Evidently, the original function and its free partner have the same expectation values when evaluated in any free matrix model for the $M_{i}$ (note that the expectation values of the products of traces appearing in the free partner factorize at large $N$ ). In general, the two expectation values will of course differ. Furthermore, if the free partner $S_{f}$ of any action $S$ is used as a trial action, it will generate a free matrix model; hence the terminology.

With the help of the above definitions, one can give the general solution of the minimization principle (10) in the space of matrix models which are free in terms of the variables $M_{i}$. The crucial observation is that, when $S_{0}$ is to be chosen from among all free actions, the original action $S$ and its free partner $S_{f}$ lead to the same minimization problem, i.e. identical right hand sides of Eq. (10), since $\langle S\rangle_{S_{0}}=\left\langle S_{f}\right\rangle_{S_{0}}$ by construction for any free $S_{0}$, as mentioned above. However, the minimization problem for $S_{f}$ is trivial to solve: Since $S_{f}$ itself describes a free matrix model, the best approximation to $S_{f}$ in the space of free actions is $S_{0}=S_{f}$ itself. Since the approximation to $S$ is governed by the same minimization problem, $S_{0}=S_{f}$ is at the same time the best free approximation to $S$.

This result provides a general solution of the minimization problem in the space of matrix models which are free in terms of the original variables $M_{i}$.

Note that the solution $S_{f}$ of the minimization problem defines a type of mean field approximation to the exact action $S$. Interaction terms in $S$ which are sensitive to angular correlations between the different matrices are replaced by terms in which each matrix couples only to selected moments of the other matrices, i.e. to some mean properties independent of the angular orientations. This is entirely analogous to the standard development of the Hartree or Hartree-Fock mean field theories from a variational principle using the space of (properly symmetrized or antisymmetrized) product states. There, in second-quantized language, the combinations in which the mean field enters the single-particle Hamiltonian are determined by Wick's theorem; here, in complete analogy, the combinations in which the moments enter the free partner are determined by the free random variable axioms as encoded in Eq. (12). In fact, (12) is nothing but Wick's theorem generalized from the usual bosonic and fermionic cases to the case of objects obeying the Cuntz algebra (this is the algebra obeyed by the Fock space operator representation of free variables, cf. Ref. [21]).

\section{Variable Transformations}

The idea of the present variational approach was stated as choosing the optimal action $S_{0}$ from among all matrix model actions which leave the matrices in terms of which $S$ is given, free with respect to one another. In this there exists an additional freedom of choice which will now be exploited, namely the choice of matrix variables in terms of which $S$ is given, i.e. in terms of which one develops the mean field approximation by constructing the free partner $S_{f}$. Up to now, only a fixed set of variables was considered - the original $M_{i}$. However, if one rewrites the action $S$ in terms of other variables,

$$
S\left(\left\{M_{i}\right\}\right)=S\left(\left\{M_{i}\left(\left\{B_{j}\right\}\right)\right\}\right)=\tilde{S}\left(\left\{B_{j}\right\}\right)
$$

and again looks for the optimal free action, now in terms of the new variables $B_{j}$, approximating $\tilde{S}$, one may find a better approximation than the one found using the variables $M_{i}$. It should be emphasized that free $B_{j}$ in general do not imply free $M_{i}$ nor vice versa. Therefore, the accessible variational space using free matrix models is in fact much larger than was apparent in the last section. By allowing different sets of variables in which to formulate the problem to be approximated, one can even include into the variation models in which the original matrices $M_{i}$ are not free with respect to one another. To formalize this idea, it is necessary to reexamine the derivation of the variational principle (10). Carrying out a variable transformation $M_{i} \rightarrow M_{i}\left(\left\{B_{j}\right\}\right)$, one has

$$
e^{-F}=\int \prod_{j} D \mu\left[B_{j}\right] e^{-N^{2} \tilde{S}\left[B_{j}\right]+\ln J\left[B_{j}\right]} \geq \exp \left[-\left(F_{0}+N^{2}\left\langle\tilde{S}-(\ln J) / N^{2}-S_{0}\right\rangle_{S_{0}}\right)\right]
$$

i.e.

$$
F \leq F_{0}+N^{2}\left\langle\tilde{S}-(\ln J) / N^{2}-S_{0}\right\rangle_{S_{0}}
$$

with the Jacobian (which must be non-vanishing to have a legitimate change of variables)

$$
J=\left|\frac{D \mu\left[M_{i}\right]}{D \mu\left[B_{j}\right]}\right|
$$

Note that this is the determinant of a $D N^{2} \times D N^{2}$ matrix, where $D$ denotes the number of matrix variables. Now, one can use the theorem of the previous section with the immediate result that the optimal free choice of $S_{0}$ in the 
new variables is the free partner of $\tilde{S}-(\ln J) / N^{2}$. Thus, while for any given set of variables, the optimal $S_{0}$ is known, one may now try to further improve the approximation (i.e. diminish the right hand side of (22) by trying different sets of variables leading to different $\tilde{S}$ and $J$.

In general, this is technically difficult, since $\ln J$ is not a multinomial expression, for which the algorithm of finding the free partner is easiest to carry out. Therefore, in this paper the treatment will be specialized to linear transformations,

$$
M_{i}=\sum_{j} c_{i j} B_{j}
$$

Then $J=\left(\operatorname{det}\left(c_{i j}\right)\right)^{N^{2}}$ is independent of the matrix variables $B_{j}$. This greatly simplifies the treatment of (22); the Jacobian does not play any role in the determination of the optimal free action $S_{0}$ for given new variables $B_{j}$ and thus the best free approximation $S_{0}$ to the given $\tilde{S}$ is the free partner $\tilde{S}_{f}$ of $\tilde{S}$. Using furthermore that $\left\langle\tilde{S}-\tilde{S}_{f}\right\rangle_{\tilde{S}_{f}}=0$, one arrives at the residual minimization problem

$$
F \leq \tilde{F}_{f}-\ln J
$$

where $\tilde{F}_{f}$ is the free energy associated with $\tilde{S}_{f}$. It now remains to try different choices of $c_{i j}$, leading to different $\tilde{S}$, $\tilde{S}_{f}$, and finally $\tilde{F}_{f}$, such as to minimize the right hand side of (25).

\section{E. Calculation of the Free Energy}

Before actually carrying out the minimization procedure, it is convenient to clarify a technical point concerning the actual evaluation of $\tilde{F}_{f}$ given $\tilde{S}_{f}$. The best way to achieve this seems to be the following. By subtracting a constant $F_{r e f}$, independent of $c_{i j}$, from both sides of (25), one arrives at the equivalent problem of minimizing $\tilde{F}_{f}-\ln J-F_{r e f}$. For $F_{r e f}$, one can choose a reference free energy, preferably of an independent matrix model with the same number of variables as $S$ and whose potential $V_{\text {ref }}$ is a polynomial of the same degree as $S$. This specification is not necessary, but convenient from the point of view of keeping the expressions one deals with in practice as regular as possible. In many applications, $V_{r e f}\left(B_{i}\right)=(1 / N) \operatorname{Tr} \sum_{i} B_{i}^{2}$ is adequate. Then one can write

$$
\begin{aligned}
\tilde{F}_{f}-F_{r e f} & =-\ln \int \prod_{j} D \mu\left[B_{j}\right] e^{-N^{2} \tilde{S}_{f}[B]}+\ln \int \prod_{j} D \mu\left[B_{j}\right] e^{-N^{2} V_{r e f}[B]} \\
& =-\left.\ln \int \prod_{j} D \mu\left[B_{j}\right] e^{-N^{2}\left(\alpha \tilde{S}_{f}[B]+(1-\alpha) V_{r e f}[B]\right)}\right|_{\alpha=0} ^{\alpha=1} \\
& =-\int_{0}^{1} d \alpha \frac{\partial}{\partial \alpha} \ln \int \prod_{j} D \mu\left[B_{j}\right] e^{-N^{2} \tilde{S}_{f}^{\prime}[B, \alpha]} \\
& =N^{2} \int_{0}^{1} d \alpha\left\langle\tilde{S}_{f}[B]-V_{r e f}[B]\right\rangle_{\tilde{S}_{f}^{\prime}}
\end{aligned}
$$

where

$$
\tilde{S}_{f}^{\prime}[B, \alpha]=\alpha \tilde{S}_{f}[B]+(1-\alpha) V_{r e f}[B]
$$

In practice, the matrix model described by $\tilde{S}_{f}^{\prime}$ is only insignificantly harder to solve than the one described by $\tilde{S}_{f}$; on the other hand, expectation values like the ones contained in (27) are very easy to evaluate and thus constitute a convenient way of evaluating the right hand side of the variational principle (25). Thus, in applications, the best formulation of the variational problem seems to be to demand minimization of

$$
\int_{0}^{1} d \alpha\left\langle\tilde{S}_{f}[B]-V_{r e f}[B]\right\rangle_{\tilde{S}_{f}^{\prime}}-(\ln J) / N^{2}
$$

as a function of the transformation matrix $c_{i j}$, where, to recapitulate, $\tilde{S}_{f}^{\prime}$ is given by (28) and $\tilde{S}_{f}$ is the free partner of $\tilde{S}$, which in turn arises from performing linear transformations on the original matrix variables $M_{i}$, i.e. 
$S\left(M_{i}\right)=S\left(c_{i j} B_{j}\right) \equiv \tilde{S}\left(B_{j}\right)$. Furthermore, $J=\left(\operatorname{det}\left(c_{i j}\right)\right)^{N^{2}}$, and $V_{\text {ref }}[B]$ is a conveniently chosen reference potential independent of $c_{i j}$ generating an independent matrix model.

As a last remark, if one has succeeded in minimizing (29) by varying the transformation $c_{i j}$, one will usually be interested in transforming back to the original variables; e.g., given the eigenvalue distributions of the matrices $B_{j}$, one may wish to extract the eigenvalue distributions of the matrices $M_{i}$. Here, the additive convolution techniques for free variables developed in [19] find fruitful application, as will become clear in the examples to be treated further below.

\section{EXAMPLES}

\section{A. Models Involving Two Classical Matrices}

The simplest type of interacting matrix model involves just two classical matrices; this provides a first testing ground for the variational approach developed in the previous sections. Without any deeper motivation, the actions

$$
S_{1}=\frac{1}{N} \operatorname{Tr}\left(M_{1}^{4}+M_{2}^{4}+g M_{1}^{2} M_{2}^{2}\right)
$$

and

$$
S_{2}=\frac{1}{N} \operatorname{Tr}\left(M_{1}^{2}+M_{2}^{2}+g M_{1} M_{2} M_{1} M_{2}\right)
$$

will be considered. The variational approximation will be compared mainly with Monte Carlo experiments, although there also already exist exact analytical solutions to some simple models of this type, see e.g. [28] in the case of $S_{2}$.

The models considered in this section are invariant under the transformation $M_{i} \rightarrow-M_{i}$, and throughout the treatment it will be assumed for simplicity that, at the large- $N$ saddle point, $\left\langle M_{i}\right\rangle=0$. Of course, it constitutes no problem in principle to work without this assumption and to verify it from the solution; this would just unnecessarily complicate the notation. In more complicated models, it may become an interesting issue whether such a reflection symmetry can be broken spontaneously.

Consider now the model described by $S_{1}$. This model will be treated here in some detail to exhibit the new techniques in practice. Later examples will receive a more cursory treatment. Allowing for a general linear transformation of variables,

$$
M_{i}=\sum_{j} c_{i j} B_{j}
$$

and inserting into $S_{1}$ to arrive at $\tilde{S}_{1}\left(B_{j}\right)$, one obtains for the free partner of the latter

$$
\tilde{S}_{1 f}=b_{1} \frac{1}{N} \operatorname{Tr} B_{1}^{4}+b_{2} \frac{1}{N} \operatorname{Tr} B_{2}^{4}+b_{12} \frac{1}{N^{2}} \operatorname{Tr} B_{1}^{2} \operatorname{Tr} B_{2}^{2}
$$

with the notations

$$
\begin{aligned}
b_{1} & =c_{11}^{4}+c_{21}^{4}+g c_{11}^{2} c_{21}^{2} \\
b_{2} & =c_{12}^{4}+c_{22}^{4}+g c_{12}^{2} c_{22}^{2} \\
b_{12} & =4\left(c_{11}^{2} c_{12}^{2}+c_{21}^{2} c_{22}^{2}\right)+g\left(c_{11}^{2} c_{22}^{2}+c_{12}^{2} c_{21}^{2}+2 c_{11} c_{12} c_{21} c_{22}\right) .
\end{aligned}
$$

Here the reflection symmetry $\left\langle B_{j}\right\rangle=0$ has been assumed to carry over from $\left\langle M_{i}\right\rangle=0$. Choosing furthermore the reference action

$$
V_{\text {ref }}=\frac{1}{N} \operatorname{Tr}\left(B_{1}^{4}+B_{2}^{4}\right)
$$

one has (cf. Eq. (28))

$$
\tilde{S}_{1 f}^{\prime}=\left(\alpha b_{1}+1-\alpha\right) \frac{1}{N} \operatorname{Tr} B_{1}^{4}+\left(\alpha b_{2}+1-\alpha\right) \frac{1}{N} \operatorname{Tr} B_{2}^{4}+\alpha b_{12} \frac{1}{N^{2}} \operatorname{Tr} B_{1}^{2} \operatorname{Tr} B_{2}^{2}
$$

Treating the last term in analogy to (15) in conjunction with $\sqrt{16})$ and $(17)$, one has to solve one-matrix problems for $B_{1}$ and $B_{2}$ with the moments $\left\langle B_{i}^{2}\right\rangle$ to be determined self-consistently. The explicit solution of the one-matrix 
model with arbitrary symmetric quartic potential is listed in Appendix A; the expressions for the second moments yield equations for the $\left\langle B_{i}^{2}\right\rangle$ which can be solved numerically for given $\alpha$ and $c_{i j}$. Then $\left\langle\tilde{S}_{1 f}-V_{\text {ref }}\right\rangle$ can be evaluated, again using the expressions for the moments given in Appendix A. Finally, evaluating the integral over $\alpha$ leads to the free energy, cf. Eq. (29), with the additional Jacobian piece $(\ln J) / N^{2}=\ln \left(c_{11} c_{22}-c_{12} c_{21}\right)$. Minimizing the free energy numerically as a function of the $c_{i j}$ yields the following result, cf. also Fig. 1: For sufficiently small $|g|$, the original variables $M_{1}$ and $M_{2}$ are the optimal ones for a free approximation, whereas for large $g$, it becomes favorable to switch variables by substituting $M_{1}=B_{1}+B_{2}$ and $M_{2}=B_{1}-B_{2}$. This change in behavior is of course simply induced by the competition between the different terms in the action. For sufficiently low $g$, the quartic part, which is already independent in the original variables $M_{1}, M_{2}$, dominates. For larger $g$, it becomes favorable to accomodate the interaction term as much as possible. The competition is essentially linear in the sense that one optimizes the treatment of either one or the other part of the action and the switch between the optimal choices of variables $M_{1}$, $M_{2}$ or $B_{1}=\left(M_{1}+M_{2}\right) / 2, B_{2}=\left(M_{1}-M_{2}\right) / 2$ happens suddenly at $g_{\mathrm{cr}}=2$; the optimal choice does not change continuously with $g$.

The authors have considered the possibility that this transition in the variational approximation may signal a large- $N$ phase transition also in the exact solution of the model, visible e.g. as a discontinuity of the derivative of the exact free energy as a function of the coupling constant $g$. However, the Monte Carlo results do not show such a transition - the expectation of $\operatorname{Tr} M_{1}^{2} M_{2}^{2}$ displays a smooth behaviour near $g_{\mathrm{cr}}$. Thus, the transition in the variational calculation seems to merely reflect a crossover between two physically different regimes rather than a genuine large- $N$ phase transition. A higher order phase transition can however not be ruled out on the basis of the data taken by the authors.

Having ascertained the optimal choice of variables, one can proceed to give the corresponding approximations to the eigenvalue distributions. For $g= \pm 1$, the original variables $M_{1}, M_{2}$ are best; the free partner of $S_{1}$ is

$$
S_{1 f}=\frac{1}{N} \operatorname{Tr}\left(M_{1}^{4}+M_{2}^{4}\right) \pm \frac{1}{N^{2}} \operatorname{Tr} M_{1}^{2} \operatorname{Tr} M_{2}^{2}
$$

Using the formulae of Appendix A to solve these models, one obtains (assuming the eigenvalue distributions of $M_{1}$ and $M_{2}$ to be identical) the consistency conditions

$$
\left\langle M_{1}^{2}\right\rangle=\left\langle M_{2}^{2}\right\rangle=x_{M}=\frac{1}{108}\left[\mp x_{M}\left(x_{M}^{2}+18\right)+\left(x_{M}^{2}+12\right)^{3 / 2}\right]
$$

solved by $x_{M}=0.334$ for $g=1$ and $x_{M}=0.476$ for $g=-1$ (the relevant solution can be picked out by using positivity of $x_{M}$, etc.). The corresponding eigenvalue distributions are given by (cf. Appendix A)

$$
\rho_{M}(\lambda)=\frac{1}{\pi}\left(2 \lambda^{2}+x_{M}+m^{2}\right) \sqrt{m^{2}-\lambda^{2}} \quad \text { with } \quad m^{2}=\frac{1}{3}\left(\sqrt{x_{M}^{2}+12}-x_{M}\right)
$$

These distributions are plotted in Figs. 2 and 3, and compared with Monte Carlo results for $10 \times 10$ matrices. In the case of $g=1$, the dependence of the Monte Carlo results on the size of the matrices is exhibited in Fig. 4. In this, as in all other cases tested, $N=10$ already seems to embody the large- $N$ asymptotic bulk behavior rather well, up to the characteristic oscillations in the eigenvalue density induced by the eigenvalue repulsion. In this respect, no deviation was observed from the well-known finite- $N$ phenomena observed in one-matrix models.

For $g=4$, on the other hand, one considers the best free approximation after the variable substitution $M_{1}=B_{1}+B_{2}$ and $M_{2}=B_{1}-B_{2}$, i.e.

$$
\tilde{S}_{1 f}=\frac{6}{N} \operatorname{Tr}\left(B_{1}^{4}+B_{2}^{4}\right)+\frac{8}{N^{2}} \operatorname{Tr} B_{1}^{2} \operatorname{Tr} B_{2}^{2}
$$

Here, the consistency condition becomes

$$
\left\langle B_{1}^{2}\right\rangle=\left\langle B_{2}^{2}\right\rangle=x_{B}=\frac{1}{486}\left[-4 x_{B}\left(16 x_{B}^{2}+27\right)+\left(16 x_{B}^{2}+18\right)^{3 / 2}\right]
$$

solved by $x_{B}=0.131$. Eqs. (38) and (39) imply that the $B_{i}$ are both governed by the quartic potential $(1 / N) \operatorname{Tr}\left(6 B_{i}^{4}+\right.$ $8 x_{B} B_{i}^{2}$ ), and the corresponding eigenvalue distribution is (cf. Appendix A)

$$
\rho_{B}(\lambda)=\frac{1}{\pi}\left(12 \lambda^{2}+8 x_{B}+6 m^{2}\right) \sqrt{m^{2}-\lambda^{2}} \quad \text { with } \quad m^{2}=\frac{1}{18}\left(\sqrt{64 x_{B}^{2}+72}-8 x_{B}\right)
$$

In order to obtain the eigenvalue distributions of the original variables $M_{1}$ and $M_{2}$ from this, one must use the free additive convolution techniques derived in [19]. In Appendix B, the convolution of two identical distributions 
governed by an arbitrary symmetric quartic potential is discussed. The resulting eigenvalue distribution $\rho_{M_{1}}=\rho_{M_{2}}$ is plotted in Fig. 5 along with the corresponding Monte Carlo results for $N=10$. Also, the comparison with the free approximation in the original variables $M_{1}, M_{2}$ is plotted. Evidently, allowing the choice of free variables to vary leads to a vastly improved approximation in this case.

In exactly the same vein as the case $g=4$ one can treat the extreme case (corresponding, up to a rescaling, to $g \rightarrow \infty)$

$$
S_{1}^{r e d}=\frac{1}{N} \operatorname{Tr} M_{1}^{2} M_{2}^{2} .
$$

Here, in the absence of any piece in the action which is free in the original variables and which even in the case of quite strong coupling $g=4$ (see above) kept the behavior of the model reasonably regular, the disagreement between the variational calculation and the exact result is rather catastrophic, cf. Fig. 6. This is not hard to understand; in the case of the action $S_{1}^{r e d}$, the variables $M_{1}, M_{2}$ can use configurations such as

$$
M_{1}=\left(\begin{array}{cc}
m_{1} & 0 \\
0 & 0
\end{array}\right) \quad M_{2}=\left(\begin{array}{cc}
0 & 0 \\
0 & m_{2}
\end{array}\right)
$$

where $m_{1}, m_{2}$ are roughly $N / 2 \times N / 2$ matrices, to preserve a low value of $S_{1}^{\text {red }}$ while realizing very large eigenvalues (together with a concentration of very small ones). This is indeed what is seen in Fig. 6. Dominance of configurations such as (42) implies a strong angular correlation between the matrices; if one rotates the above matrices with respect to one another, e.g. such that the eigenvalues are arbitrarily reordered, $S_{1}^{r e d}$ will in general take a very large value. Such angular correlations are completely lost in the free partner $S_{1 f}^{r e d}=\left(1 / N^{2}\right) \operatorname{Tr} M_{1}^{2} \operatorname{Tr} M_{2}^{2}$ and evidently even the best choice of linearly transformed variables $M_{1}=B_{1}+B_{2}$ and $M_{2}=B_{1}-B_{2}$, though incorporating some angular correlations between $M_{1}$ and $M_{2}$, cannot do much to alleviate this problem. It is however possible that a nonlinear change of variables exists for which the difficulty will be satisfactorily removed. The authors did not explore this direction. The dismal failure of the variational approximation in this case thus does not come as a complete surprise. Rather, it is gratifying to see how well the variational approximation still works in the previous, not quite as pathological, example described by $S_{1}$ with the strong coupling of $g=4$. One striking feature of the Monte Carlo eigenvalue distribution in the case of $S_{1}^{r e d}$ is the absence of the characteristic finite- $N$ oscillations observed in all other cases. This can be understood as follows: Usually, the potential confines the eigenvalues to a compact domain and, due to the eigenvalue repulsion, they tend to be equidistant. This (fluctuating) lattice has a favored equilibrium position, leading to the oscillations in the eigenvalue density. However, when the action allows the eigenvalues to spread over the entire real axis, the correlations induced by the eigenvalue repulsion become insignificant and the oscillations in the eigenvalue density disappearf?

Apart from plotting the eigenvalue distributions, which essentially contain the information about all the moments of the individual matrices, one can test mixed correlators for freeness properties. In particular, if the matrices $M_{1}$ and $M_{2}$ are free with respect to each other, then there are e.g. the following relations between correlators, following from the axioms of freeness:

$$
\begin{aligned}
C_{1} \equiv\left\langle M_{1}^{2} M_{2}^{2}\right\rangle & =\left\langle M_{1}^{2}\right\rangle\left\langle M_{2}^{2}\right\rangle \equiv C_{1 f} \\
C_{2} \equiv\left\langle M_{1}^{4} M_{2}^{4}\right\rangle & =\left\langle M_{1}^{4}\right\rangle\left\langle M_{2}^{4}\right\rangle \equiv C_{2 f} \\
C_{3} \equiv\left\langle M_{1}^{2} M_{2}^{2} M_{1}^{2} M_{2}^{2}\right\rangle & =\left\langle M_{1}^{4}\right\rangle\left\langle M_{2}^{2}\right\rangle^{2}+\left\langle M_{1}^{2}\right\rangle^{2}\left\langle M_{2}^{4}\right\rangle-\left\langle M_{1}^{2}\right\rangle^{2}\left\langle M_{2}^{2}\right\rangle^{2} \equiv C_{3 f}
\end{aligned}
$$

In the Monte Carlo calculations, both sides of these equations were sampled in order to give a measure for the deviation from freeness (i.e. strength of angular correlations) contained in the exact models. The results are tabulated in Table 1 for different values of $g$ in $S_{1}$. Note that for $S_{1}^{r e d}$, the moments of the exact distribution diverge due to the evidently too slow fall-off of the eigenvalue distribution for large eigenvalues, making such a comparison impossible.

\begin{tabular}{|c||c|c||c|c||c|c||c|c|}
\hline$g$ & $\left\langle M_{i}^{2}\right\rangle$ & $\left\langle M_{i}^{4}\right\rangle$ & $C_{1}$ & $C_{1 f}$ & $C_{2}$ & $C_{2 f}$ & $C_{3}$ & $C_{3 f}$ \\
\hline \hline-1 & .483 & .377 & .254 & .233 & .171 & .142 & .150 & .121 \\
\hline 1 & .336 & .197 & .106 & .113 & .0339 & .0387 & .0272 & .0317 \\
\hline 4 & .267 & .133 & .0585 & .0713 & .0116 & .0177 & .0085 & .0139 \\
\hline
\end{tabular}

Table 1 : Mixed correlators, Eqs. (43), for the model $S_{1}$ compared to the predictions for free $M_{i}$

\footnotetext{
${ }^{2}$ The authors are indebted to U.-J. Wiese for this remark.
} 
One observes how, for growing $g$, the interaction term introduces stronger angular correlations, evident in the stronger deviations from free predictions. Now, for sufficiently large $g$, e.g. $g=4$, the variational principle asserts that a better approximation is obtained with a new set of mutually free variables $B_{i}$. Using this new set of free variables, of course also the predictions for mixed correlators, derived in (43) for free $M_{i}$, change. This shall be illustrated here for the correlator $\left\langle M_{1}^{2} M_{2}^{2}\right\rangle$. Inserting $M_{1}=B_{1}+B_{2}$ and $M_{2}=B_{1}-B_{2}$, using the axioms of freeness on $B_{1}$ and $B_{2}$ together with $\left\langle B_{i}\right\rangle=0$, and then substituting back $B_{1}=\left(M_{1}+M_{2}\right) / 2$ and $B_{2}=\left(M_{1}-M_{2}\right) / 2$, one obtains

$$
\begin{aligned}
\left\langle M_{1}^{2} M_{2}^{2}\right\rangle & =\left\langle B_{1}^{4}+B_{2}^{4}\right\rangle=\frac{1}{8}\left\langle M_{1}^{4}+M_{2}^{4}+4 M_{1}^{2} M_{2}^{2}+2 M_{1} M_{2} M_{1} M_{2}\right\rangle \\
\left\langle M_{1} M_{2} M_{1} M_{2}\right\rangle & =\left\langle B_{1}^{4}+B_{2}^{4}\right\rangle-4\left\langle B_{1}^{2}\right\rangle\left\langle B_{2}^{2}\right\rangle=\left\langle M_{1}^{2} M_{2}^{2}\right\rangle-\frac{1}{4}\left(\left\langle M_{1}^{2}+M_{2}^{2}\right\rangle^{2}-4\left\langle M_{1} M_{2}\right\rangle^{2}\right) \\
\left\langle M_{1} M_{2}\right\rangle & =\left\langle B_{1}^{2}\right\rangle-\left\langle B_{2}^{2}\right\rangle=0
\end{aligned}
$$

where in the last line, identical distributions for the $B_{i}$ were assumed. Putting these relations together yields $\left\langle M_{1}^{2} M_{2}^{2}\right\rangle$ in terms of the individual moments,

$$
\left\langle M_{1}^{2} M_{2}^{2}\right\rangle=\frac{1}{2}\left\langle M_{1}^{4}+M_{2}^{4}\right\rangle-\frac{1}{4}\left\langle M_{1}^{2}+M_{2}^{2}\right\rangle^{2}
$$

In the case $g=4$, the right hand side, using the individual moments from the Monte Carlo experiment, takes the value .0617 , which indeed is closer to the exact value for $\left\langle M_{1}^{2} M_{2}^{2}\right\rangle \equiv C_{1}$ than the prediction using free variables $M_{i}$ quoted in Table 1.

Consider now the model described by

$$
S_{2}=\frac{1}{N} \operatorname{Tr}\left(M_{1}^{2}+M_{2}^{2}+g M_{1} M_{2} M_{1} M_{2}\right) .
$$

After performing a general linear transformation as in (30), one arrives at the free partner

$$
\begin{aligned}
\tilde{S}_{2 f}=\left(c_{11}^{2}+c_{21}^{2}\right) & \frac{1}{N} \operatorname{Tr} B_{1}^{2}+\left(c_{12}^{2}+c_{22}^{2}\right) \frac{1}{N} \operatorname{Tr} B_{2}^{2}+g c_{11}^{2} c_{21}^{2} \frac{1}{N} \operatorname{Tr} B_{1}^{4}+ \\
& +g c_{12}^{2} c_{22}^{2} \frac{1}{N} \operatorname{Tr} B_{2}^{4}+4 g c_{11} c_{12} c_{21} c_{22} \frac{1}{N^{2}} \operatorname{Tr} B_{1}^{2} \operatorname{Tr} B_{2}^{2} .
\end{aligned}
$$

Evidently, from the point of view of the variational approximation, this model is slightly simpler than the one considered above. Since the noninteracting part of $S_{2}$ is invariant under linear transformations of the variables and subsequent construction of the free partner, up to trivial variable rescalings (note also that, as before, $\left\langle B_{i}\right\rangle=0$ was used in deriving (48)), the choice of variables will always be such as to best accomodate the interacting term, for any coupling $g$. Carrying out the calculation of the free energy in complete analogy to the calculation for $S_{1}$, this turns out to be the choice $M_{1}=B_{1}+B_{2}, M_{2}=B_{1}-B_{2}$, corresponding to the free partner

$$
\tilde{S}_{2 f}=\frac{1}{N} \operatorname{Tr}\left(g B_{1}^{4}+g B_{2}^{4}+2 B_{1}^{2}+2 B_{2}^{2}\right)-\frac{4 g}{N^{2}} \operatorname{Tr} B_{1}^{2} \operatorname{Tr} B_{2}^{2}
$$

The corresponding consistency condition is

$$
\left\langle B_{1}^{2}\right\rangle=\left\langle B_{2}^{2}\right\rangle=x_{B}=\frac{1}{108 g^{2}}\left[-\left(2-4 g x_{B}\right)^{3}-18 g\left(2-4 g x_{B}\right)+\left(\left(2-4 g x_{B}\right)^{2}+12 g\right)^{3 / 2}\right]
$$

From the resulting eigenvalue distributions of the matrices $B_{i}$ one again obtains the eigenvalue distributions of the original variables $M_{i}$ by additive convolution. The result for $g=2 / 5$, leading to $x_{B}=.2522$ in (50), is displayed in Fig. [7, compared with the Monte Carlo result for $N=40$. Also, various correlators are tabulated in Table 2.

\begin{tabular}{|c||c|c||c|c||c|c||c|c|}
\hline$g$ & $\left\langle M_{i}^{2}\right\rangle$ & $\left\langle M_{i}^{4}\right\rangle$ & $C_{1}$ & $C_{1 f}$ & $C_{2}$ & $C_{2 f}$ & $C_{3}$ & $C_{3 f}$ \\
\hline \hline $2 / 5$ & .53 & .56 & .30 & .28 & .37 & .32 & .28 & .24 \\
\hline
\end{tabular}

Table 2 : Mixed correlators, Eqs. (43), for the model $S_{2}$, compared to the predictions for free $M_{i}$ 
The exact eigenvalue distribution turns out to be surprisingly similar to the semicircle of radius $\sqrt{2}$ obtained at $g=0$. This is well reproduced by the variational calculation despite a quite strong dependence on $g$ of the individual coefficients in $\tilde{S}_{2 f}$. The effects of the $g$-dependence of the quadratic and quartic coefficients nearly cancel in the complete potential, leading to a quite stable eigenvalue distribution.

Turning to the mixed correlators in Table 2, one does not observe a strong deviation from free behavior of the $M_{i}$. In this case, there is almost no room for improvement by assuming free $B_{i}$ instead of $M_{i}$, leading to equation (47) for $\left\langle M_{1}^{2} M_{2}^{2}\right\rangle$. Indeed, one here obtains for the right hand side of (47) the value .28, the same as $C_{1 f}$ in Table 2 .

The reader may wonder why only the relatively small value $g=2 / 5$ was displayed in the comparison above. In fact, the model described by $S_{2}$ becomes unstable for too large values of $|g|$. Note that the product of two hermitian matrices $M_{1} M_{2}$ is in general not hermitian; therefore, the interaction term, proportional to $\left(M_{1} M_{2}\right)^{2}$, is not bounded from below even for positive $g$. Strictly speaking, the model is unstable for all $g$, but for sufficiently small $g$, there is effectively a barrier posed by the independent part of the action preventing the system from spilling over to the region of large eigenvalues where the interaction will allow eigenvalues to grow without bound. A glimpse of this instability (for negative $g$, namely setting in at $g=-4 / 9$ ) was already given in [28]. Here, instead the instability at positive $g$ was investigated in slightly more detail. According to Monte Carlo simulations at $N=10,20$, and 40, the critical coupling lies in the interval $g \in[0.4,0.45]$. On the other hand, the variational approximation in the best basis, described by the action (49), is stable up to $g=2$. Beyond this point, there is no solution to the consistency condition (50). The authors also checked that there is no two-cut solution to (49) above $g=2$ (note that (50) is valid only for one-cut solutions). Thus, the variational approximation does qualitatively capture the phase structure of the model described by $S_{2}$, albeit with a badly overestimated critical coupling. In this respect, the choice of variables $B_{1}=\left(M_{1}+M_{2}\right) / 2$ and $B_{2}=\left(M_{1}-M_{2}\right) / 2$ represents a drastic improvement over the original variables $M_{i}$. In terms of the latter, the free counterpart of $S_{2}$ is simply $S_{2 f}=(1 / N) \operatorname{Tr}\left(M_{1}^{2}+M_{2}^{2}\right)$, which entirely misses the unstable phase of the model (in fact, all the dependence on the coupling $g$ ).

\section{B. Classical Constant Matrices in Many Dimensions}

Consider a model of $D$ classical matrices with an action of the type

$$
S_{D}=\frac{1}{D-1} \sum_{i \neq j}^{D} S\left(M_{i}, M_{j}\right)
$$

Note the scaling of this action with $D$, which is necessary to retain a meaningful balance between it and the eigenvalue repulsion originating in the Haar measure of the $M_{i}$. Before proceeding, a comment is in order regarding the special form of (51) and the interpretation of $D$ as the number of space-time dimensions. In (51), each matrix degree of freedom interacts with all others in the same way. If one wishes to attach physical meaning to the matrices as degrees of freedom living in space-time, this occurs most naturally when all the degrees of freedom are attached to the same space-time point. Such a model may become relevant if one has managed to decouple the different space-time points of a $D$-dimensional large- $N$ field theory, e.g. as the result of an Eguchi-Kawai reduction (for a review, see Ref. [29]). Now, in view of the scaling of the individual terms in (51), one might hope that the detailed angular correlations between pairs of matrices become unimportant at large $D$ and a free approximation becomes exact. This argument can be made rigorous e.g. in the Kazakov-Migdal model, where the leading large- $D$ behavior is described by a free matrix model [30]. The proof however depends on the specific link variable structure of the Kazakov-Migdal model. In order to test this idea more generally, the models described by

$$
S_{D 2}=\frac{1}{D-1} \sum_{i \neq j}^{D} \frac{1}{N} \operatorname{Tr} M_{i}^{2} M_{j}^{2} \quad \text { and } \quad S_{D 4}=\frac{1}{D-1} \sum_{i \neq j}^{D} \frac{1}{N} \operatorname{Tr} M_{i}^{4} M_{j}^{4}
$$

were investigated in the present framework for different $D$. The reader is reminded that in the case of $S_{D 2}$ for $D=2$, the free variational approximation failed miserably (cf. previous section). In analyzing the models (52) in the free variational approximation, the authors did not treat different choices of free variables; for simplicity, only the best

\footnotetext{
${ }^{3}$ Presumably, the model described by $S_{1}$ will display a similar instability when the coupling $g$ becomes too negative. This was not investigated further by the authors.
} 
free approximation in the original variables $M_{i}$ was considered. Then the best free approximation is easily derived: The free counterparts of (52) are

$$
S_{D 2 f}=\frac{1}{D-1} \sum_{i \neq j}^{D} \frac{1}{N^{2}} \operatorname{Tr} M_{i}^{2} \operatorname{Tr} M_{j}^{2} \quad \text { and } \quad S_{D 4 f}=\frac{1}{D-1} \sum_{i \neq j}^{D} \frac{1}{N^{2}} \operatorname{Tr} M_{i}^{4} \operatorname{Tr} M_{j}^{4}
$$

and thus each variable $M_{i}$ is described by the action

$$
\bar{S}_{D 2}\left(M_{i}\right)=2 x_{D 2} \frac{1}{N} \operatorname{Tr} M_{i}^{2} \quad \text { or } \quad \bar{S}_{D 4}\left(M_{i}\right)=2 x_{D 4} \frac{1}{N} \operatorname{Tr} M_{i}^{4}
$$

independent of $D$, with $x_{D 2}=\left\langle M_{i}^{2}\right\rangle$ and $x_{D 4}=\left\langle M_{i}^{4}\right\rangle$ to be determined self-consistently. One obtains $x_{D 2}=1 / 2$ and $x_{D 4}=1 / \sqrt{8}$ and therefore the eigenvalue distributions

$$
\rho_{2}(\lambda)=\frac{1}{\pi} \sqrt{2-\lambda^{2}} \quad \text { and } \quad \rho_{4}(\lambda)=\frac{1}{\pi}\left(\sqrt{2} \lambda^{2}+\sqrt{\frac{\sqrt{8}}{3}}\right) \sqrt{\sqrt{\frac{2 \sqrt{8}}{3}}-\lambda^{2}}
$$

Comparing this with the results of Monte Carlo experiments, cf. Figs. 8 and 9, the correspondence is quite satisfying already at $D=10$ (the simulations were carried out for $N=10$, some checks for $N=20$ did not reveal any significant differences). Also, comparing mixed correlators of two matrices (without loss of generality, $M_{1}$ and $M_{2}$ ) with free predictions (cf. equations (43)) in the Monte Carlo experiments, one obtains good agreement for $D=10$, cf. Table 3 .

\begin{tabular}{|c||c|c||c|c||c|c||c|c|}
\hline & $\left\langle M_{i}^{2}\right\rangle$ & $\left\langle M_{i}^{4}\right\rangle$ & $C_{1}$ & $C_{1 f}$ & $C_{2}$ & $C_{2 f}$ & $C_{3}$ & $C_{3 f}$ \\
\hline \hline$S_{D 2}, D=3$ & .62 & 1.00 & .25 & .38 & .33 & 1.00 & .18 & .62 \\
\hline$S_{D 2}, D=10$ & .51 & .53 & .25 & .26 & .25 & .28 & .18 & .21 \\
\hline \hline$S_{D 4}, D=3$ & .49 & .46 & .20 & .25 & .12 & .21 & .09 & .16 \\
\hline$S_{D 4}, D=10$ & .46 & .37 & .21 & .22 & .13 & .13 & .11 & .11 \\
\hline
\end{tabular}

Table 3 : Mixed correlators, Eq. (43), for the models (52), and predictions for free $M_{i}$

As mentioned above, this agreement does not come unexpected. However, while in the cases considered here the Monte Carlo solution seemed to converge towards the free approximation at large $D$, it seems doubtful that this should be true in general. On the one hand, a solution with angular correlations, say with angles restricted to a certain sector as compared with some fixed reference matrix, costs a weight $\exp \left(-\alpha N^{2} D\right)$ in the partition sum (there are $O\left(N^{2} D\right)$ angles, and the coefficient $\alpha$ depends on the details of the angular distributions). On the other hand, the interaction itself is also scaled to be of order $O\left(N^{2} D\right)$. Therefore, there should be a genuine competition between the two terms, the former favoring freeness and the latter preventing it.

\section{Quantum Mechanics of One Matrix}

For a wide class of potentials, the quantum mechanical ground state of a single large- $N$ matrix can be solved for exactly [9]. This is achieved by working directly with continuous (Euclidean) time and mapping the problem to a noninteracting fermion problem. If one discretizes time as in the original definition of the path integral, the problem can be interpreted in terms of many interacting classical matrices. In this form, the quantum mechanics of one matrix is hard to solve exactly; on the other hand, it becomes amenable to the variational method dealt with here. Interest in such a discretized form has arisen in recent applications to quantum gravity, [31. For definiteness, the model described by

$$
S_{Q 1}=\frac{1}{N} \operatorname{Tr}\left(\sum_{i=0}^{L-1}\left(M_{i+1}-M_{i}\right)^{2}+g M_{i}^{4}\right)
$$

will be considered here, where $L$ is the number of time slices, and $M_{0}$ is identified with $M_{L}$, i.e. periodic boundary conditions are posited. Generically, there is a competition between two different interaction terms: The nearestneighbor coupling and the potential term, which acts instantaneously. In order to best accomodate the former in 
a free variational approximation, one will want to change matrix variables to a Fourier basis, which decouples the kinetic part of the action. On the other hand, the potential term will prefer the original variables. Thus, in the present treatment, not a general linear change of variables will be considered, but only the aforementioned two discrete choices; the variational minimization principle will decide which choice is preferred. The Fourier basis is defined as

$$
M_{i}=\sum_{j} U_{i j} B_{j}
$$

with ( $L$ is for simplicity taken to be even)

$$
U=\sqrt{\frac{2}{L}}\left(\begin{array}{cccccccc}
0 & \cdots & 0 & 1 / \sqrt{2} & 1 & \cdots & 1 & 1 / \sqrt{2} \\
\sin k_{L / 2-1} & \cdots & \sin k_{1} & 1 / \sqrt{2} & \cos k_{1} & \cdots & \cos k_{L / 2-1} & -1 / \sqrt{2} \\
\sin 2 k_{L / 2-1} & \cdots & \sin 2 k_{1} & 1 / \sqrt{2} & \cos 2 k_{1} & \cdots & \cos 2 k_{L / 2-1} & 1 / \sqrt{2} \\
\sin 3 k_{L / 2-1} & \cdots & \sin 3 k_{1} & 1 / \sqrt{2} & \cos 3 k_{1} & \cdots & \cos 3 k_{L / 2-1} & -1 / \sqrt{2} \\
\vdots & \ddots & \vdots & \vdots & \vdots & \ddots & \vdots & \vdots \\
\sin (L-1) k_{L / 2-1} & \cdots & \sin (L-1) k_{1} & 1 / \sqrt{2} & \cos (L-1) k_{1} & \cdots & \cos (L-1) k_{L / 2-1} & -1 / \sqrt{2}
\end{array}\right)
$$

Here, the wavevectors are $k_{j}=2 \pi j / L$; note that $U$ is orthogonal and therefore the Jacobian term in the free energy (29) gives no contribution for the Fourier choice of variables. For convenience in notation, the index $j$ labeling the variables $B_{j}$ in (57), i.e. the columns in (58), will be taken to run from $-L / 2+1$ to $L / 2$.

In the Fourier basis, the kinetic part of the action (56) is exactly diagonalized and coincides with its free partner

$$
\tilde{S}_{Q 1}^{k i n}\left(B_{i}\right)=\frac{2}{N} \operatorname{Tr} \sum_{j=-L / 2+1}^{L / 2}\left(1-\cos k_{j}\right) B_{j}^{2}=\tilde{S}_{Q 1 f}^{k i n}\left(B_{i}\right)
$$

On the other hand, the potential part in the new variables is

$$
\tilde{S}_{Q 1}^{p o t}\left(B_{i}\right)=\frac{g}{N} \operatorname{Tr} \sum_{i} \sum_{j k l m} U_{i j} B_{j} U_{i k} B_{k} U_{i l} B_{l} U_{i m} B_{m}
$$

Assuming as before $\left\langle B_{i}\right\rangle=0$, the only terms which will give a contribution to the free partner are ones in which the four indices $j, k, l, m$ all take the same value or pairwise two different values. Thus, one has the free partner

$$
\tilde{S}_{Q 1 f}^{p o t}\left(B_{i}\right)=g\left[4 \sum_{j<k} \frac{1}{N^{2}} \operatorname{Tr} B_{j}^{2} \operatorname{Tr} B_{k}^{2}\left(\sum_{i} U_{i j}^{2} U_{i k}^{2}\right)+\sum_{j} \frac{1}{N} \operatorname{Tr} B_{j}^{4}\left(\sum_{i} U_{i j}^{4}\right)\right]
$$

By explicit calculation, $\sum_{i} U_{i j}^{4}$ is of order $O(1 / L)$ and therefore the quartic term can be dropped for a large number of time slices $L$. On the other hand, again by explicit calculation,

$$
\sum_{i=0}^{L-1} U_{i j}^{2} U_{i k}^{2}=\frac{1}{L} \quad \text { for all } j, k
$$

and therefore one finally has for the free partner of the action (56) in the Fourier variables $B_{i}$,

$$
\tilde{S}_{Q 1 f}\left(B_{i}\right)=2 \sum_{i=-L / 2+1}^{L / 2}\left(1-\cos k_{i}\right) \frac{1}{N} \operatorname{Tr} B_{i}^{2}+\frac{4 g}{L} \sum_{i<j=-L / 2+1}^{L / 2} \frac{1}{N^{2}} \operatorname{Tr} B_{i}^{2} \operatorname{Tr} B_{j}^{2}
$$

On the other hand, the free partner of (56) in the original variables is

$$
S_{Q 1 f}\left(M_{i}\right)=\frac{1}{N} \operatorname{Tr} \sum_{i=0}^{L-1}\left(2 M_{i}^{2}+g M_{i}^{4}\right)
$$

again assuming $\left\langle M_{i}\right\rangle=0$. Here, the potential term is treated exactly, whereas the nearest-neighbor coupling has been completely truncated. 
The free models are now easily solved. For the purpose of calculating the free energies, the reference action $V_{\text {ref }}\left(C_{i}\right)=(1 / N) \operatorname{Tr} \sum_{i} C_{i}^{2}$ will be used; then one considers (cf. Eq. 28))

$$
S_{Q 1 f}^{\prime}\left(M_{i}\right)=\frac{1}{N} \operatorname{Tr} \sum_{i}\left[(1+\alpha) M_{i}^{2}+\alpha g M_{i}^{4}\right]
$$

for the original variables and

$$
\tilde{S}_{Q 1 f}^{\prime}\left(B_{i}\right)=\sum_{i}\left(1+\alpha-2 \alpha \cos k_{i}\right) \frac{1}{N} \operatorname{Tr} B_{i}^{2}+\frac{4 \alpha g}{L} \sum_{i<j} \frac{1}{N^{2}} \operatorname{Tr} B_{i}^{2} \operatorname{Tr} B_{j}^{2}
$$

for the Fourier variables. In the latter, introducing the abbreviation

$$
x_{B}=\frac{2}{L}\left\langle\sum_{i} B_{i}^{2}\right\rangle
$$

one has semicircular distributions for the matrix variables $B_{i}$ of radius squared

$$
r_{i}^{2}=\frac{2}{1+\alpha-2 \alpha \cos k_{i}+\alpha g x_{B}}
$$

implying second moments $\left\langle B_{i}^{2}\right\rangle=r_{i}^{2} / 4$, which self-consistently determines $x_{B}$ :

$$
\begin{aligned}
x_{B}=\frac{2}{L}\left\langle\sum_{i} B_{i}^{2}\right\rangle & =\frac{1}{L} \sum_{i} \frac{1}{1+\alpha-2 \alpha \cos (2 \pi i / L)+\alpha g x_{B}} \\
& \stackrel{L \rightarrow \infty}{\longrightarrow} \int_{-1 / 2}^{1 / 2} d k \frac{1}{1+\alpha+\alpha g x_{B}-2 \alpha \cos 2 \pi k} \\
& =\frac{1}{\sqrt{\left(\alpha+\alpha g x_{B}+1\right)^{2}-4 \alpha^{2}}}
\end{aligned}
$$

or,

$$
x_{B}^{2}\left(\alpha+\alpha g x_{B}+1\right)^{2}-4 \alpha^{2} x_{B}^{2}-1=0
$$

The free energy is then

$$
\begin{aligned}
F_{B} & =\int_{0}^{1} d \alpha \sum_{i} \frac{1}{2} \frac{2-2 \cos (2 \pi i / L)+g x_{B}-1}{1+\alpha-2 \alpha \cos (2 \pi i / L)+\alpha g x_{B}} \\
& \stackrel{L \rightarrow \infty}{\longrightarrow} L \int_{0}^{1} d \alpha \int_{-1 / 2}^{1 / 2} d k \frac{1}{2} \frac{2-2 \cos 2 \pi k+g x_{B}-1}{1+\alpha-2 \alpha \cos 2 \pi k+\alpha g x_{B}} \\
& =L \int_{0}^{1} d \alpha \frac{1}{2 \alpha}\left[1-\frac{1}{\sqrt{\left(\alpha+\alpha g x_{B}+1\right)^{2}-4 \alpha^{2}}}\right]
\end{aligned}
$$

which must be calculated numerically (remember that at every $\alpha, x_{B}$ is determined by the equation (70)).

On the other hand, in the decoupled basis, one has the quartic action (65), identical and independent for all the $M_{i}$. Using Appendix $\mathrm{A}$, the resulting moments are

$$
\begin{aligned}
& \left\langle M^{2}\right\rangle=\frac{1}{108 \alpha^{2} g^{2}}\left[-(1+\alpha)^{3}-18 \alpha g(1+\alpha)+\left((1+\alpha)^{2}+12 \alpha g\right)^{3 / 2}\right] \\
& \left\langle M^{4}\right\rangle=\frac{1}{216 \alpha^{3} g^{3}}\left[(1+\alpha)^{4}+18 \alpha g(1+\alpha)^{2}+54 \alpha^{2} g^{2}-(1+\alpha)\left((1+\alpha)^{2}+12 \alpha g\right)^{3 / 2}\right]
\end{aligned}
$$

and, inserting in the free energy,

$$
\begin{aligned}
F_{M} & =\int_{0}^{1} d \alpha 2 L\left\langle M^{2}\right\rangle+L g\left\langle M^{4}\right\rangle-L\left\langle M^{2}\right\rangle \\
& =\frac{L}{108 g^{2}}\left[-4-36 g-\frac{81 g^{2}}{2}+(4+30 g) \sqrt{1+3 g}+54 g^{2} \ln (1+\sqrt{1+3 g})\right]
\end{aligned}
$$


Numerically, $F_{M}=F_{B}$ at $g=1.2306$. For smaller $g$, the Fourier basis provides the better approximation, for larger $g$ the original choice of variables does. It has been argued in [31] that matrix models such as the one considered here exhibit a Kosterlitz-Thouless transition at some value of the lattice spacing separating the two regimes where the matrix chain behaves more like a collection of independent sites and the one where it supports spin wave type of modes. The variational approach presented here, while giving no details of the transition region, is capable of capturing these two different possible regimes.

It remains to give the eigenvalue distributions in the two regimes. In the original variables, described by the action $S_{Q 1 f}\left(M_{i}\right)$, cf. equation (64), one immediately has, using Appendix A,

$$
\rho_{M}(\lambda)=\frac{1}{\pi}\left(2 g \lambda^{2}+2+m^{2} g\right) \sqrt{m^{2}-\lambda^{2}} \quad \text { with } m^{2}=\frac{2}{3 g}(\sqrt{1+3 g}-1)
$$

for all the variables $M_{i}$. In the Fourier case, one must convolute the semicircular distributions of the $B_{i}$ to obtain the distributions of the $M_{i}$. It is well known [19] that the semicircular distributions are closed under additive convolution and that the square radii add up to the square radius of the resulting semicircle. I.e.,

$$
r_{M_{i}}^{2}=\sum_{j} U_{i j}^{2} r_{B_{j}}^{2}
$$

Inserting (68) for $\alpha=1$ and (58), one obtains identical radii for all the $M_{i}$,

$$
\begin{aligned}
r_{M_{i}}^{2} & =\frac{1}{L} \sum_{j=-L / 2+1}^{L / 2} \frac{2}{2-2 \cos (2 \pi j / L)+g x_{B}} \\
& \stackrel{L \rightarrow \infty}{\longrightarrow} \int_{-1 / 2}^{1 / 2} d k \frac{2}{2-2 \cos 2 \pi k+g x_{B}} \\
& =\frac{2}{\sqrt{4 g x_{B}+g^{2} x_{B}^{2}}} \\
& =2 x_{B}
\end{aligned}
$$

where in the last line it has been used that $x_{B}=(2 / L)\left\langle\sum_{i} B_{i}^{2}\right\rangle$ solves (cf. (70) for $\left.\alpha=1\right)$

$$
4 g x_{B}^{3}+g^{2} x_{B}^{4}=1
$$

The corresponding semicircular distribution for the $M_{i}$ is then

$$
\rho_{M}(\lambda)=\frac{1}{x_{B} \pi} \sqrt{2 x_{B}-\lambda^{2}}
$$

The variational approximation derived above should now be compared to Monte Carlo experiments and the exact results known about this model. In Figs. $10-12$ Monte Carlo results for $L=10$ and $N=10$ are exhibited together with variational results at different couplings $g$. Checks with $N=20$ and $L=20$ revealed no significant differences. One indeed observes, as already borne out by the calculation of the free energies, that the Fourier basis is preferable in a weak potential and the original basis is favored by a strong potential. This is corroborated by the Monte Carlo evaluation of correlators between matrices at neighboring time slices, cf. Table 4.

\begin{tabular}{|c||c|c||c|c||c|c||c|c|}
\hline$g$ & $\left\langle M_{i}^{2}\right\rangle$ & $\left\langle M_{i}^{4}\right\rangle$ & $C_{1}$ & $C_{1 f}$ & $C_{2}$ & $C_{2 f}$ & $C_{3}$ & $C_{3 f}$ \\
\hline \hline .1 & .55 & .57 & .41 & .31 & .59 & .33 & .52 & .26 \\
\hline 1 & .24 & .11 & .066 & .059 & .015 & .012 & .014 & .0093 \\
\hline 10 & .098 & .017 & .0099 & .0097 & .00031 & .00029 & .00025 & .00024 \\
\hline
\end{tabular}

Table 4 : Mixed correlators, Eqs. (43), between matrices $M_{i}$ at neighboring time slices and predictions for free $M_{i}$

At large coupling, neighboring matrices are to a very good approximation free, meaning the original decoupled basis gives a good description. By contrast, at low $g$, there are significant angular correlations induced by the nearestneighbor coupling. These angular correlations are washed out by the fluctuations when one considers matrices far apart on the temporal lattice, cf. Table 5 . There is virtually no deviation from free behavior between distant matrices even at low values of $g$. 


\begin{tabular}{|c||c|c||c|c||c|c||c|c|}
\hline$g$ & $\left\langle M_{i}^{2}\right\rangle$ & $\left\langle M_{i}^{4}\right\rangle$ & $C_{1}$ & $C_{1 f}$ & $C_{2}$ & $C_{2 f}$ & $C_{3}$ & $C_{3 f}$ \\
\hline \hline .1 & .55 & .57 & .31 & .31 & .35 & .33 & .28 & .26 \\
\hline 1 & .24 & .11 & .059 & .059 & .012 & .012 & .0093 & .0093 \\
\hline 10 & .098 & .017 & .0097 & .0097 & .00029 & .00029 & .00024 & .00024 \\
\hline
\end{tabular}

Table 5 : Mixed correlators, Eqs. (43), between matrices $M_{i}$ separated by half of the total length of the temporal lattice, and predictions for free $M_{i}$

In order to make contact with physical (still Euclidean) time, one should write the discretized action of the quantum one-matrix model as

$$
S_{Q 1}^{\prime}=\frac{1}{N} \operatorname{Tr}\left(\sum_{i=0}^{L-1} \frac{\left(M_{i+1}^{\prime}-M_{i}^{\prime}\right)^{2}}{2} \frac{L}{T}+\tilde{g} \frac{T}{L}\left(M_{i}^{\prime}\right)^{4}\right)
$$

where $T$ is now the length of the circle in time direction. Rescaling $M_{i}^{\prime}=M_{i} \sqrt{2 T / L}$, one regains the form (56) of the action, with the identification $g=4 \tilde{g} T^{3} / L^{3}$. Therefore, $g$ behaves like the third power of the lattice spacing.

In the continuum limit, the ground state distribution of the eigenvalues of the quantum mechanical one-matrix model has been found analytically in [9]. This can now be compared to the variational solution. According to the correspondence established above, the limit of vanishing lattice spacing corresponds to vanishing $g$; therefore the Fourier basis is the preferable one. The eigenvalue distribution for the rescaled variables $M_{i}^{\prime}$ is (cf. equation (79))

$$
\rho_{M^{\prime}}(\lambda)=\sqrt{\frac{L}{2 T}} \rho_{M}\left(\sqrt{\frac{L}{2 T}} \lambda\right)=\sqrt{\frac{L}{2 T}} \frac{1}{x_{B} \pi} \sqrt{2 x_{B}-\frac{L}{2 T} \lambda^{2}}
$$

and $x_{B}$ is determined by (cf. equation $(78)$ )

$$
16 \tilde{g} \frac{T^{3}}{L^{3}} x_{B}^{3}+16 \tilde{g}^{2} \frac{T^{6}}{L^{6}} x_{B}^{4}=1
$$

which for large $L$ is solved by

$$
x_{B}=\frac{L}{T}(16 \tilde{g})^{-1 / 3}+O(1)
$$

Therefore

$$
\rho_{M^{\prime}}(\lambda)=\frac{2}{\pi}\left(\frac{\tilde{g}}{4}\right)^{1 / 3} \sqrt{\left(\frac{4}{\tilde{g}}\right)^{1 / 3}-\lambda^{2}}
$$

Note that there is no $T$-dependence left in the continuum limit. By contrast, the exact solution is [9]

$$
\rho_{M^{\prime}}^{\text {exact }}(\lambda)=\frac{1}{\pi} \sqrt{2 \epsilon-2 \tilde{g} \lambda^{4}} \quad \text { with } \quad \epsilon^{3}=81 \pi^{6} \tilde{g}\left(\frac{1}{\Gamma(1 / 4)}\right)^{8}
$$

determined by the normalization condition. The two solutions are compared for $\tilde{g}=1$ in Fig. 13 .

\section{Quantum Mechanics of Two Matrices}

While the case of one quantum mechanical matrix discussed in the previous section is amenable to exact treatment [9] due to its special form, an analogous solution is not possible once more than one quantum mechanical matrix is involved. On the other hand, the approximate variational approach, using discretized time, is easily generalized to the case of more than one matrix. Here, a commutator-type interaction will be considered, as it is especially interesting from the point of view of applications to Yang-Mills theories:

$$
S_{Q 2}=\frac{1}{N} \operatorname{Tr}\left(\sum_{i=0}^{L-1}\left(M_{1, i+1}-M_{1, i}\right)^{2}+\left(M_{2, i+1}-M_{2, i}\right)^{2}+g\left(i\left[M_{1, i}, M_{2, i}\right]\right)^{2}\right)
$$


where the first index labels the two different matrix variables and the second one the different time slices. Again, only the original choice of variables will be compared to a Fourier basis (cf. (58)) using the variational criterion.

Note that the action $S_{Q 2}$ is invariant under simultaneous shifts of all the matrices by a multiple of the unit matrix. This trivial freedom should be removed in order to obtain stable Monte Carlo results; otherwise, the system just performs a random walk in the trace of the matrices. Thus, the $M_{n, i}$ here are constrained to be traceless, i.e. the condition $\left\langle M_{n, i}\right\rangle=0$, assumed to be realized dynamically in prior examples, is enforced by hand.

The free partner to (86) in the original basis is

$$
S_{Q 2 f}\left(M_{n, i}\right)=\sum_{i=0}^{L-1} \frac{1}{N} \operatorname{Tr}\left(2 M_{1, i}^{2}+2 M_{2, i}^{2}\right)+\frac{2 g}{N^{2}} \operatorname{Tr} M_{1, i}^{2} \operatorname{Tr} M_{2, i}^{2}
$$

(using $\left\langle M_{1, i}\right\rangle=\left\langle M_{2, i}\right\rangle=0$ ). On the other hand, in the Fourier basis

$$
M_{n, i}=\sum_{j} U_{i j} B_{n, j}
$$

with $U$ as in (58), the kinetic part becomes

$$
\tilde{S}_{Q 2}^{k i n}\left(B_{n, i}\right)=\tilde{S}_{Q 2 f}^{k i n}\left(B_{n, i}\right)=\frac{2}{N} \operatorname{Tr} \sum_{j=-L / 2+1}^{L / 2}\left(1-\cos k_{j}\right)\left(B_{1, j}^{2}+B_{2, j}^{2}\right)
$$

The potential part on the other hand becomes

$$
-\frac{g}{N} \operatorname{Tr} \sum_{i}\left[M_{1, i}, M_{2, i}\right]^{2}=\frac{2 g}{N} \operatorname{Tr} \sum_{i} \sum_{j k l m} U_{i j} U_{i k} U_{i l} U_{i m}\left(B_{1, j} B_{1, k} B_{2, l} B_{2, m}-B_{1, j} B_{2, k} B_{1, l} B_{2, m}\right)
$$

Using again the assumption $\left\langle B_{n, i}\right\rangle=0$, the second term in the round brackets on the right hand side never gives a contribution to the free partner, whereas the first term only gives a contribution if $j=k$ and $l=m$. Thus one has

$$
\tilde{S}_{Q 2 f}^{p o t}\left(B_{n, i}\right)=\frac{2 g}{N^{2}} \sum_{j, k} \operatorname{Tr} B_{1, j}^{2} \operatorname{Tr} B_{2, k}^{2} \sum_{i} U_{i j} U_{i j} U_{i k} U_{i k}=\frac{2 g}{N^{2} L} \sum_{j, k} \operatorname{Tr} B_{1, j}^{2} \operatorname{Tr} B_{2, k}^{2}
$$

Now one can again easily solve the free models. For the purpose of calculating the free energies, the reference action

$$
V_{\text {ref }}\left(C_{n, i}\right)=\frac{1}{N} \operatorname{Tr} \sum_{i}\left(C_{1, i}^{2}+C_{2, i}^{2}\right)
$$

is convenient; then one has to solve the models

$$
S_{Q 2 f}^{\prime}\left(M_{n, i}\right)=\sum_{i=0}^{L-1} \frac{1+\alpha}{N} \operatorname{Tr}\left(M_{1, i}^{2}+M_{2, i}^{2}\right)+\frac{2 \alpha g}{N^{2}} \operatorname{Tr} M_{1, i}^{2} \operatorname{Tr} M_{2, i}^{2}
$$

for the original variables $M_{n, i}$ and

$$
\tilde{S}_{Q 2 f}^{\prime}\left(B_{n, i}\right)=\sum_{j}\left(1+\alpha-2 \alpha \cos k_{j}\right) \frac{1}{N} \operatorname{Tr}\left(B_{1, j}^{2}+B_{2, j}^{2}\right)+\frac{2 g \alpha}{N^{2} L} \sum_{j, k} \operatorname{Tr} B_{1, j}^{2} \operatorname{Tr} B_{2, k}^{2}
$$

for the Fourier variables $B_{n, i}$. Starting with (93), abbreviating $\left\langle M_{n, i}^{2}\right\rangle=x_{M}$, one obtains identical semicircular distributions for all matrix variables with radius squared

$$
r^{2}=\frac{2}{\alpha+2 \alpha g x_{M}+1}
$$

whereupon the consistency condition determining $x_{M}$ becomes

$$
x_{M}=\frac{r^{2}}{4}=\frac{1}{2} \frac{1}{\alpha+2 \alpha g x_{M}+1}
$$


solved by

$$
x_{M}=\frac{1}{4 \alpha g}\left(\sqrt{(1+\alpha)^{2}+4 g \alpha}-(1+\alpha)\right)
$$

The free energy then is given by

$$
F_{M}=L \int_{0}^{1} d \alpha\left(4 x_{M}+2 g x_{M}^{2}-2 x_{M}\right)
$$

On the other hand, in the Fourier case (94), abbreviating

$$
\left\langle\frac{2}{L} \sum_{j} B_{1, j}^{2}\right\rangle=\left\langle\frac{2}{L} \sum_{j} B_{2, j}^{2}\right\rangle=x_{B}
$$

one notices that the variables $B_{1, j}$ and $B_{2, j}$ are controlled by exactly the same potential as the variables $B_{j}$ in the case of the quantum mechanical one-matrix model discussed in the previous section. In particular, one has the consistency condition

$$
x_{B}^{2}\left(\alpha+\alpha g x_{B}+1\right)^{2}-4 \alpha^{2} x_{B}^{2}-1=0
$$

and the second moments

$$
\left\langle B_{1, j}^{2}\right\rangle=\left\langle B_{2, j}^{2}\right\rangle=\frac{1}{2} \frac{1}{1+\alpha-2 \alpha \cos k_{j}+\alpha g x_{B}}
$$

The free energy now is

$$
F_{B}=L \int_{0}^{1} d \alpha\left[2 x_{B}-\frac{1}{L} \sum_{j=-L / 2+1}^{L / 2} \frac{2 \cos (2 \pi j / L)}{1+\alpha-2 \alpha \cos (2 \pi j / L)+\alpha g x_{B}}+\frac{g x_{B}^{2}}{2}-x_{B}\right]
$$

Numerical evaluation of the two free energies yields $F_{B} \leq F_{M}$ for all $g$, i.e. the Fourier basis is always preferable over the original basis. While this is to be expected at small $g$, it is not necessarily an indication that the Fourier basis represents a particularly good approximation for large $g$ as well: Rather, working in the original basis still truncates the interaction term rather badly at every time slice (cf. equation (87)). This is different from the one-matrix case, where assuming the original variables to be free merely implied decoupling variables at different time slices, which certainly should become exact for large coupling $g$. In the two-matrix case, by contrast, there is still an additional truncation at every time slice which prevents convergence to the exact problem at large $g$. In light of this, it is not so surprising that the Fourier basis is preferable for all $g$. Nevertheless, in Figs. 14 16, this basis appears to provide quite a satisfactory description even for $g=10$; the agreement with Monte Carlo results $(N=10$ and $L=10$ were used in the latter) is not much worse at $g=10$ than at $g=1 / 10$.

Also in the correlators, cf. Tables 6 and 7, one observes that the angular correlations involving the same matrix variable at different time slices are stronger than the angular correlations between the two different matrix variables at a fixed time, up to quite high $g$. At $g=10$, these two correlations seem to be roughly equally strong. This again corroborates the result that using the Fourier basis, which treats the nearest-neighbor coupling exactly, provides a good approximation for a large range of $g$.

\begin{tabular}{|c||c|c||c|c||c|c||c|c|}
\hline$g$ & $\left\langle M_{n, i}^{2}\right\rangle$ & $\left\langle M_{n, i}^{4}\right\rangle$ & $C_{1}$ & $C_{1 f}$ & $C_{2}$ & $C_{2 f}$ & $C_{3}$ & $C_{3 f}$ \\
\hline \hline .1 & .86 & 1.5 & 1.2 & .75 & 5.6 & 2.3 & 5.1 & 1.7 \\
\hline 1 & .38 & .29 & .19 & .14 & .16 & .084 & .13 & .063 \\
\hline 10 & .18 & .068 & .038 & .033 & .0064 & .0047 & .005 & .0034 \\
\hline
\end{tabular}

Table 6 : Mixed correlators, Eqs. (43), between matrices $M_{n, i}$ for one fixed $n$ at neighboring time slices $i$ and predictions for free $M_{n, i}$

\begin{tabular}{|c||c|c||c|c||c|c||c|c|}
\hline$g$ & $\left\langle M_{n, i}^{2}\right\rangle$ & $\left\langle M_{n, i}^{4}\right\rangle$ & $C_{1}$ & $C_{1 f}$ & $C_{2}$ & $C_{2 f}$ & $C_{3}$ & $C_{3 f}$ \\
\hline \hline .1 & .86 & 1.5 & .66 & .75 & 1.7 & 2.3 & 1.3 & 1.7 \\
\hline 1 & .38 & .29 & .12 & .14 & .059 & .084 & .043 & .063 \\
\hline 10 & .18 & .068 & .027 & .033 & .0027 & .0047 & .002 & .0034 \\
\hline
\end{tabular}


Table 7 : Mixed correlators between the matrices $M_{1, i}$ and $M_{2, i}$ at a fixed time slice $i$ and predictions for free $M_{1, i}$ $M_{2, i}$.

Ultimately, though, one would expect a local treatment, which assumes different time slices to be free with respect to one another, to provide better agreement at high $g$ if it manages to well approximate the commutator interaction. This is precisely what is not achieved if the original variables are assumed to be free. On the contrary, a nontrivial mixing of the variables $M_{1, i}$ and $M_{2, i}$ at every time slice is necessary such as to better accomodate the commutatortype interaction in (86). Note that linear combinations of $M_{1, i}$ and $M_{2, i}$ will not effect any improvement, since the commutator is invariant under such linear transformations (up to trivial rescalings of the variables). It is disappointing that precisely this phenomenologically very interesting type of interaction term seems particularly resistent to free approximation combined with linear transformations. Here, it seems that using (technically more complicated) nonlinear variable transformations must be contemplated in order to capture the essential angular correlations. Note also that the commutator term in the action on its own does not confine the eigenvalue distributions of the involved variables to a compact support, even if one enforces tracelessness; the eigenvalues can become arbitrarily large if the matrices are located in the regions of configuration space where they commute. This pathology however seems to disappear (according to Monte Carlo experiments carried out by the authors) when more than two matrices are involved (i.e. a higher-dimensional Yang-Mills type of action). Presumably there, the regions of configuration space where all the matrices commute are too small compared with the whole space such that entropy suppresses configurations with arbitrarily large eigenvalues.

\section{SUMMARY}

In this work, a new variational approach to interacting large- $N$ multi-matrix models was developed. The partition function was approximated using the variational principle $F \leq F_{0}+\left\langle S-S_{0}\right\rangle_{S_{0}}$ with $S_{0}$ initially taken from the space of all matrix models which are free in the set of variables the original interacting problem is given in. It turned out to be possible to give a general solution to this variational problem for a fixed set of matrix variables in terms of the concept of the "free partner" introduced by the authors. The free partner defines a type of mean field approximation to the original action and is constructed using the axioms of freeness in a fashion analogous to the construction of more conventional mean field theories for fermionic or bosonic particles using Wick's theorem. The freeness axioms constitute the analog of Wick's theorem for objects obeying the Cuntz algebra, which is the algebra obeyed by the Fock space operator representation of free random variables [21].

However, the variational approach presented here goes beyond simply acting as a device to derive a mean field theory. Above, the variational space was characterized as being the space of all matrix models which are free in the set of variables the original interacting problem is given in. This implies that one can considerably enlarge the variational space by first allowing for a change of variables in the original problem and only then varying over all matrix models which are free in the variables one has settled for. In this way one includes into the variation models which are not free in the original variables. Every set of variables defines a different mean field theory given by the corresponding free partner. The variational principle not only allows to derive these mean field theories, but further allows to decide which out of a set of mean field theories provides the best approximation to the exact problem.

A number of examples were considered in order to assess the accuracy of the variational method; the variational results were compared with Monte Carlo simulations as well as analytical results, where available. To begin with, classical two-matrix models described by actions with at most quartic terms were investigated allowing for a general linear transformation of matrix variables. Impressive agreement was observed, except in the case of the action $S_{1}^{\text {red }}$, Eq. (41). The authors did not attempt to improve the approximation by allowing for nonlinear variable transformations, which may relieve the discrepancy. Classical models with a large number of matrices were considered in which all matrices pairwise interact in the same way. Such models e.g. become relevant when one has managed to decouple the different space-time points of a large- $N$ matrix field theory in a large number of dimensions $D$, e.g. in the framework of an Eguchi-Kawai reduction. Among the models considered was one which reduces to $S_{1}^{\text {red }}$ for $D=2$. It turned out that the quality of the variational approximation using only the original set of variables improves as $D$ rises.

The variational approximation was also considered for the problems of a single and two coupled matrix chains simulating path integrals for one and two quantum matrices. Two discrete choices of free variables, namely the original variables and a Fourier basis, were considered. In the single chain case, as expected, for small (large) lattice spacing, the Fourier (original) basis provided the better approximation. In the continuum limit of the one-matrix case, good agreement was achieved with the available analytical solution. For two matrix chains coupled via a commutatortype interaction, the Fourier basis provided a better approximation for all couplings; it also compares quite favorably with the exact Monte Carlo results for a large range of coupling constants. However, for very large coupling, one would expect a local basis, constructed such as to capture the angular correlations introduced by the commutator interaction, 
to ultimately be more appropriate. Here, again it seemed that technically more involved nonlinear transformations must be contemplated in order to achieve further progress.

\section{APPENDIX A: SOLUTION OF THE ONE-MATRIX MODEL IN A SYMMETRIC QUARTIC POTENTIAL}

Following [9], 32] the one-cut eigenvalue distribution $\rho$ resulting from a hermitian large- $N$ one-matrix model in a quartic potential $V(x)=a_{4} x^{4}+a_{2} x^{2}$ is most easily found by considering the resolvent,

$$
G(z):=\int_{-\infty}^{\infty} d \lambda \frac{\rho(\lambda)}{z-\lambda}=\frac{1}{2} V^{\prime}(z)-Q(z) \sqrt{z^{2}-m^{2}}
$$

where $Q(z)$ is a polynomial. $Q(z)$, along with $m^{2}$, which defines the support of $\rho$, is fixed by comparing coefficients in the asymptotic behavior of $G$,

$$
G(z) \stackrel{z \rightarrow \infty}{\longrightarrow} \frac{1}{z}+\sum_{n=1}^{\infty} \frac{c_{n}}{z^{n+1}}
$$

where $c_{n}$ is the $n$-th moment of the distribution $\rho$. One obtains

$$
Q(z)=2 a_{4} z^{2}+a_{2}+m^{2} a_{4} \quad m^{2}=\frac{1}{3 a_{4}}\left(\sqrt{a_{2}^{2}+12 a_{4}}-a_{2}\right)
$$

Then one can also immediately read off

$$
\rho(\lambda)=-\frac{1}{\pi} \operatorname{Im} G(\lambda+i \epsilon)=\frac{1}{\pi} Q(\lambda) \sqrt{m^{2}-\lambda^{2}}
$$

(where $\epsilon \rightarrow 0$ ), and by expanding $G$ in $1 / z$,

$$
\begin{aligned}
& c_{2}=\frac{1}{108 a_{4}^{2}}\left[-a_{2}^{3}-18 a_{4} a_{2}+\left(a_{2}^{2}+12 a_{4}\right)^{3 / 2}\right] \\
& c_{4}=\frac{1}{216 a_{4}^{3}}\left[a_{2}^{4}+18 a_{2}^{2} a_{4}+54 a_{4}^{2}-a_{2}\left(a_{2}^{2}+12 a_{4}\right)^{3 / 2}\right]
\end{aligned}
$$

When $a_{4}=0$, these expressions simplify to

$$
\begin{aligned}
\rho(\lambda) & =\frac{1}{\pi} a_{2} \sqrt{\left(2 / a_{2}\right)-\lambda^{2}} \\
c_{2} & =\frac{1}{2 a_{2}} \\
c_{4} & =\frac{1}{2 a_{2}^{2}}
\end{aligned}
$$

i.e. the second moment of the semicircular distribution is one fourth of its square radius.

In complete analogy, one can derive the two-cut solution in the quartic potential. Then, $G$ has the form

$$
G(z)=2 a_{4} z^{3}+a_{2} z-q \sqrt{\left(z^{2}-m_{+}^{2}\right)\left(z^{2}-m_{-}^{2}\right) z^{2}}
$$

from which one again obtains by comparing coefficients in the asymptotic expansion

$$
q=2 a_{4} \quad m_{ \pm}^{2}=-\frac{a_{2}}{2 a_{4}} \pm \frac{1}{\sqrt{a_{4}}}
$$

One can immediately read off the eigenvalue distribution, and the second moment e.g. becomes

$$
c_{2}=\frac{a_{4}}{8}\left(m_{+}^{6}-m_{+}^{4} m_{-}^{2}-m_{+}^{2} m_{-}^{4}+m_{-}^{6}\right)
$$




\section{APPENDIX B: FREE ADDITIVE CONVOLUTION OF TWO IDENTICAL DISTRIBUTIONS GOVERNED BY A QUARTIC POTENTIAL}

In order to additively convolute the eigenvalue distributions of two matrices which are free with respect to one another, i.e. to obtain $\rho_{B_{1}+B_{2}}$ given $\rho_{B_{1}}$ and $\rho_{B_{2}}$, one should go to the corresponding R-transforms [19. These are defined as follows: Find the resolvents $G_{B_{1}}$ and $G_{B_{2}}$ as defined in Appendix A; then the R-transforms are given by $R(y)=G^{-1}(y)-1 / y$ (here $G^{-1}$ denotes the inverse function, $\left.G^{-1}(G(x))=x\right)$. The R-transforms behave additively, i.e. $R_{B_{1}+B_{2}}=R_{B_{1}}+R_{B_{2}}$. In this sense they are the free analogs of the logarithm of the Fourier transform for probability distributions of ordinary commuting random variables.

In the case of matrices governed by a quartic potential $V(x)=a_{4} x^{4}+a_{2} x^{2}$, the resolvent has already been given explicitly in Appendix A; denoting $G(z) \equiv y$, one has that $z \equiv G^{-1}(y)$ satisfies

$$
y=2 a_{4} z^{3}+a_{2} z-\left(2 a_{4} z^{2}+a_{2}+m^{2} a_{4}\right) \sqrt{z^{2}-m^{2}}
$$

where $m^{2}$ was defined in Appendix A. Isolating the square root term and squaring the resulting equation leads to a third order equation for $z$,

$$
4 a_{4} y z^{3}-4 a_{4} z^{2}+2 a_{2} y z-y^{2}-m^{2}\left(a_{2}+m^{2} a_{4}\right)^{2}=0
$$

It is not necessary to solve this explicitly, since the case of interest here is the case of two identical distributions, i.e. identical R-transforms. Then one has

$$
w \equiv G_{B_{1}+B_{2}}^{-1}(y)=R_{B_{1}+B_{2}}+\frac{1}{y}=R_{B_{1}}+R_{B_{2}}+\frac{1}{y}=G_{B_{1}}^{-1}(y)+G_{B_{2}}^{-1}(y)-\frac{1}{y}=2 z-\frac{1}{y}
$$

implying

$$
z=\frac{1}{2}\left(w+\frac{1}{y}\right)
$$

However, the equation satisfied by $z$ is known, equation (B2), which by inserting (B4) leads to the equation satisfied by $w \equiv G_{B_{1}+B_{2}}^{-1}(y)$. Since one is actually interested in $y \equiv G_{B_{1}+B_{2}}(w)$, one can solve directly for $y$ by rearranging (B2) in conjunction with (B4) as a polynomial in $y$,

$$
2 y^{4}-y^{3}\left(a_{4} w^{3}+2 a_{2} w\right)-y^{2}\left(a_{4} w^{2}+2 a_{2}-2 m^{2}\left(a_{2}+m^{2} a_{4}\right)^{2}\right)+y a_{4} w+a_{4}=0
$$

Rather than obtaining a cumbersome analytical solution of (B5), the relevant solution was tracked numerically. This is best done in small steps in along the positive real $w$-axis starting from the known asymptotic behavior $y=1 / w+O\left(w^{-3}\right)$ and using the positivity of the eigenvalue distribution $\rho_{B_{1}+B_{2}}$. The latter is essentially given by the imaginary part of $y$, namely $\rho_{B_{1}+B_{2}}(\lambda)=-\operatorname{Im} G_{B_{1}+B_{2}}(\lambda+i \epsilon) / \pi$.

[1] G.'t Hooft, Nucl. Phys. B 72 (1974) 461.

[2] G.'t Hooft, Nucl. Phys. B 75 (1974) 461.

[3] S.Coleman, 1/N, in: Aspects of Symmetry (Cambridge University Press, Cambridge, 1985).

[4] C.G.Callan, N.Coote and D.J.Gross, Phys. Rev. D 13 (1976) 1649.

[5] M.B.Einhorn, Phys. Rev. D 14 (1976) 3451.

[6] E.Witten, Nucl. Phys. B 160 (1979) 57, Nucl. Phys. B 223 (1983) 433.

[7] G.S.Adkins, C.R.Nappi and E.Witten, Nucl. Phys. B 228 (1983) 552.

[8] Y.M.Makeenko and A.A.Migdal, Phys. Lett. B 88 (1979) 135; A.A.Migdal, Phys. Rep. 102 (1983) 199.

[9] E.Brézin, C.Itzykson, G.Parisi and J.-B.Zuber, Comm. Math. Phys. 59 (1978) 35.

[10] C.Itzykson and J.B.Zuber, J. Math. Phys. 21 (1980) 411.

[11] M.L.Mehta, Random Matrices (Academic Press, New York, 1991).

[12] The Large-N Expansion in Quantum Field Theory and Statistical Physics: From Spin Systems to 2-Dimensional Gravity, eds. E.Brézin and S.R.Wadia (World Scientific, Singapore, 1993).

[13] B.L.Altshuler and B.D.Simons, in: Proceedings of Les Houches Summer School, Session LXI, 1994, eds. E.Akkermans, G.Montambaux, J.-L.Pichard and J.Zinn-Justin. 
[14] J.Ambjørn, J.Jurkiewicz and Yu.M.Makeenko, Phys. Lett. B 251 (1990) 517.

[15] E.Brézin and A.Zee, Nucl. Phys. B 402 [FS] (1993) 613.

[16] E.Brézin and A.Zee, Phys. Rev. E 49 (1994) 2588; E.Brézin, S.Hikami and A.Zee, Phys. Rev. E 51 (1995) 5442.

[17] V.A.Kazakov, M.Staudacher and T.Wynter, preprints hep-th/9502132, hep-th/9506174, hep-th/9601069

[18] V.A.Kazakov and A.A.Migdal, Nucl.Phys. B 397 (1993) 214.

[19] D.V.Voiculescu, K.J.Dykema and A.Nica, Free Random Variables (Am. Math. Soc., Providence, RI, 1992).

[20] I.M.Singer, unpublished, and in: Functional Analysis on the Eve of the 21st Century, in Honor of I.M.Gelfand, eds. S.Gindikin, J.Lepowsky and R.L.Wilson, Progress in Mathematics, Vol. 131 (Birkhäuser, Boston, 1995).

[21] R.Gopakumar and D.J.Gross, Nucl. Phys. B 451 (1995) 379.

[22] M.R.Douglas, Nucl. Phys. B 41 (Proc. Suppl.) (1995) 66.

[23] A.Zee, Nucl. Phys. B 474 [FS] (1996) 726.

[24] I.Ya.Aref'eva and I.V.Volovich, preprint hep-th/9510210.

[25] P.Neu and R.Speicher, J. Phys. A 28 (1995) L79; J. Stat. Phys. 80 (1995) 1279.

[26] R.P.Feynman and A.R.Hibbs, Quantum Mechanics and Path Integrals (McGraw-Hill, New York, 1965).

[27] S.Das, A.Dhar, A.Sengupta and S.Wadia, Mod. Phys. Lett. A 5 (1990) 1041.

[28] L.Chekhov and C.Kristjansen, preprint hep-th/9605013.

[29] S.R.Das, Rev. Mod. Phys. 59 (1987) 235.

[30] Y.Makeenko, preprint hep-th/9512211.

[31] D.J.Gross and I.Klebanov, Nucl. Phys. B 344 (1990) 475.

[32] N.I.Akhiezer and I.M.Glazman, Theory of Linear Operators in Hilbert Space, Vol.I (Frederick Ungar, New York, 1963).

FIG. 1. Free energies of free variational approximations to $S_{1}$ as a function of the coupling constant $g$ for different sets of linearly transformed variables $M_{i}=\sum_{j} c_{i j} B_{j}$, where $c_{i j}$ is orthogonal, $c_{i j}=\sigma_{i j}^{3} \cos \phi+\sigma_{i j}^{1} \sin \phi$ (and $\sigma^{k}$ are the Pauli matrices). Note how all trajectories cross at $g_{\mathrm{cr}}=2 ; \phi=0$ provides the best approximation below $g_{\mathrm{cr}}$ and $\phi=\pi / 4$ above $g_{\mathrm{cr}}$. Trajectories generated by nonorthogonal $c_{i j}$ always lie higher and are not displayed.

FIG. 2. Eigenvalue distribution $\rho(\lambda)$ generated by $S_{1}$ at $g=-1$. Data points: Monte Carlo result for $N=10$. Solid line: Best free variational approximation.

FIG. 3. Eigenvalue distribution $\rho(\lambda)$ generated by $S_{1}$ at $g=1$. Data points: Monte Carlo result for $N=10$. Solid line: Best free variational approximation.

FIG. 4. Eigenvalue distribution $\rho(\lambda)$ generated by $S_{1}$ at $g=1$ in Monte Carlo simulations for $N=20$ (solid line), $N=10$ (dotted), $N=3$ (dashed).

FIG. 5. Eigenvalue distribution $\rho(\lambda)$ generated by $S_{1}$ at $g=4$. Data points: Monte Carlo result for $N=10$. Solid line: Best free variational approximation, namely in the variables $M_{1}+M_{2}, M_{1}-M_{2}$. Dotted: Free approximation in the original variables $M_{1}, M_{2}$.

FIG. 6. Eigenvalue distribution $\rho(\lambda)$ generated by $S_{1}^{r e d}$. Data points: Monte Carlo result for $N=10$. Solid line: Best free variational approximation.

FIG. 7. Eigenvalue distribution $\rho(\lambda)$ generated by $S_{2}$ at $g=2 / 5$. Data points: Monte Carlo result for $N=40$. Solid line: Best free variational approximation.

FIG. 8. Eigenvalue distribution $\rho(\lambda)$ generated by $S_{D 2}$. Monte Carlo results for $N=10$ are given in the cases $D=2$ (dash-dotted), $D=3$ (dashed) and $D=10$ (dotted). Solid line: Free variational approximation in the original variables $M_{i}$.

FIG. 9. Eigenvalue distribution $\rho(\lambda)$ generated by $S_{D 4}$. Monte Carlo results for $N=10$ are given in the cases $D=2$ (dash-dotted), $D=3$ (dashed) and $D=10$ (dotted). Solid line: Free variational approximation in the original variables $M_{i}$. 
FIG. 10. Eigenvalue distribution $\rho(\lambda)$ generated by $S_{Q 1}$ at $g=0.1$. Data points: Monte Carlo result for $N=10$ and $L=10$. Solid line: Free variational approximation in the Fourier basis. Dotted: Free variational approximation in the original basis. The former is the better approximation of the two.

FIG. 11. Eigenvalue distribution $\rho(\lambda)$ generated by $S_{Q 1}$ at $g=1$. Data points: Monte Carlo result for $N=10$ and $L=10$. Solid line: Free variational approximation in the Fourier basis. Dotted: Free variational approximation in the original basis. The former is the better approximation of the two.

FIG. 12. Eigenvalue distribution $\rho(\lambda)$ generated by $S_{Q 1}$ at $g=10$. Data points: Monte Carlo result for $N=10$ and $L=10$. Solid line: Free variational approximation in the Fourier basis. Dotted: Free variational approximation in the original basis. The latter is the better approximation of the two.

FIG. 13. Eigenvalue distribution $\rho(\lambda)$ generated by $S_{Q 1}$ in the continuum limit, namely for $\tilde{g}=1$ (for the definition of $\tilde{g}$, see text). Dotted: Exact result. Solid line: Free variational approximation in the Fourier basis.

FIG. 14. Eigenvalue distribution $\rho(\lambda)$ generated by $S_{Q 2}$ at $g=0.1$. Data points: Monte Carlo result for $N=10$ and $L=10$. Solid line: Free variational approximation in the Fourier basis.

FIG. 15. Eigenvalue distribution $\rho(\lambda)$ generated by $S_{Q 2}$ at $g=1$. Data points: Monte Carlo result for $N=10$ and $L=10$. Solid line: Free variational approximation in the Fourier basis.

FIG. 16. Eigenvalue distribution $\rho(\lambda)$ generated by $S_{Q 2}$ at $g=10$. Data points: Monte Carlo result for $N=10$ and $L=10$. Solid line: Free variational approximation in the Fourier basis. 


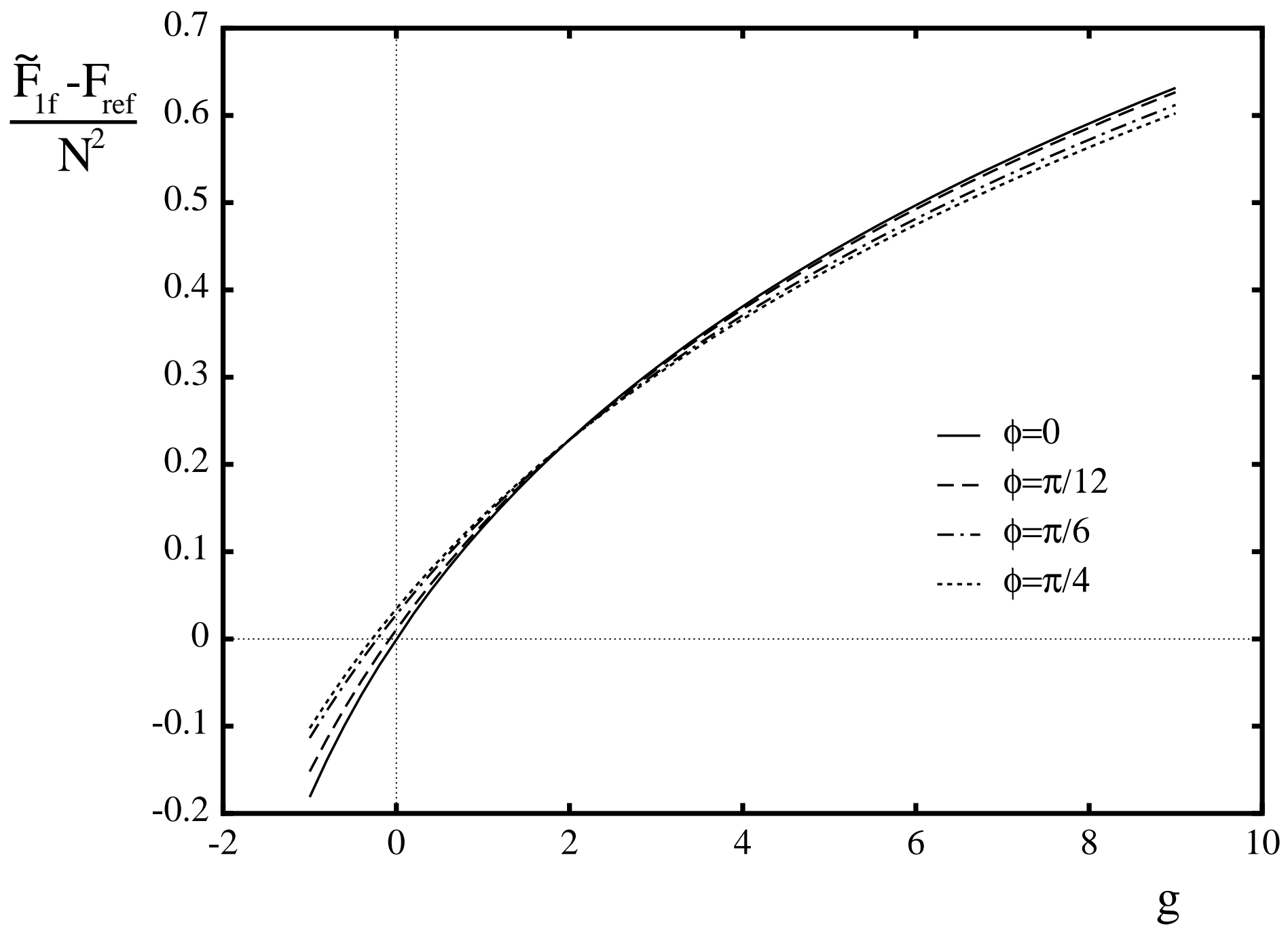

Figure 1 


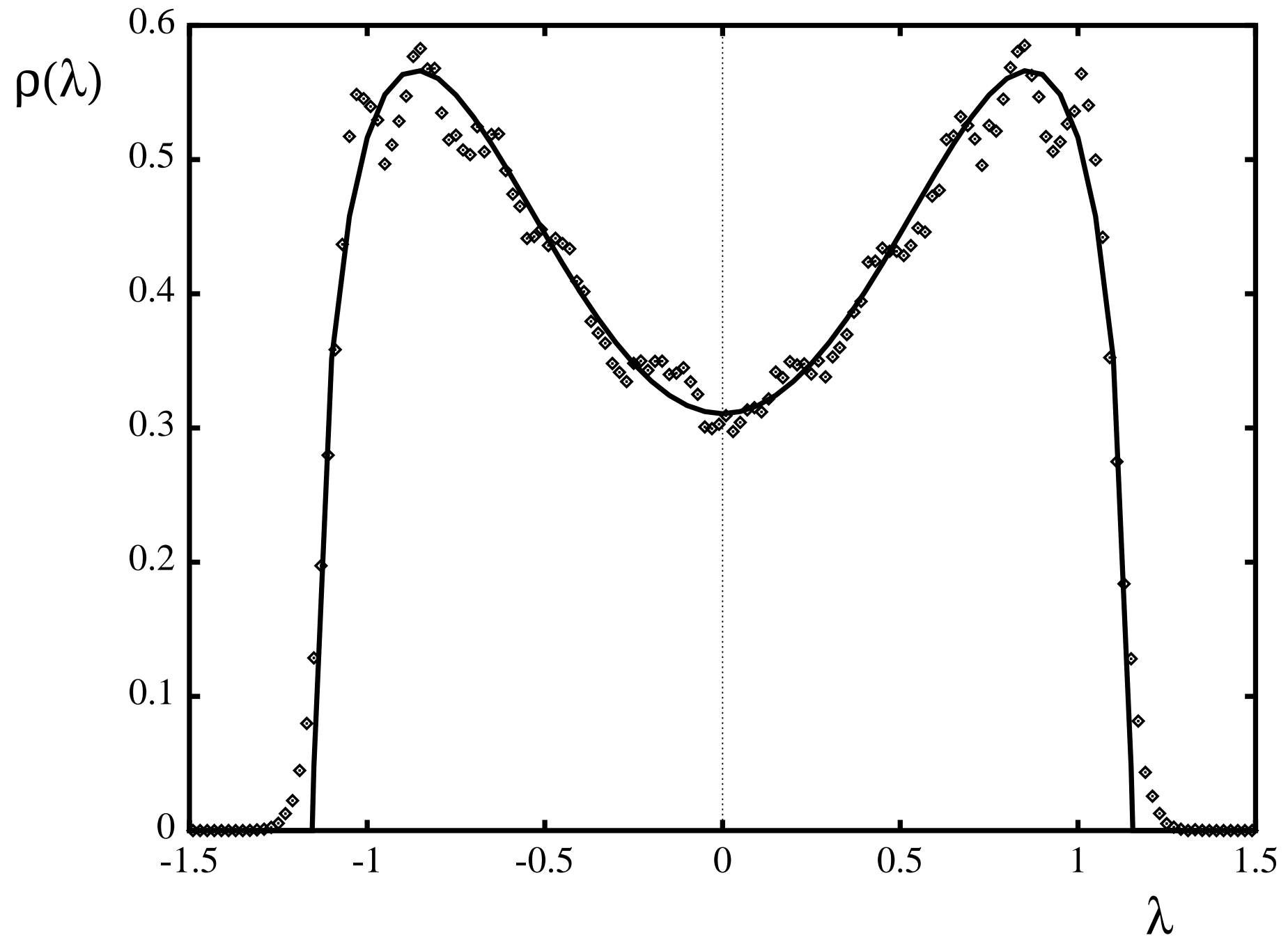

Figure 2 


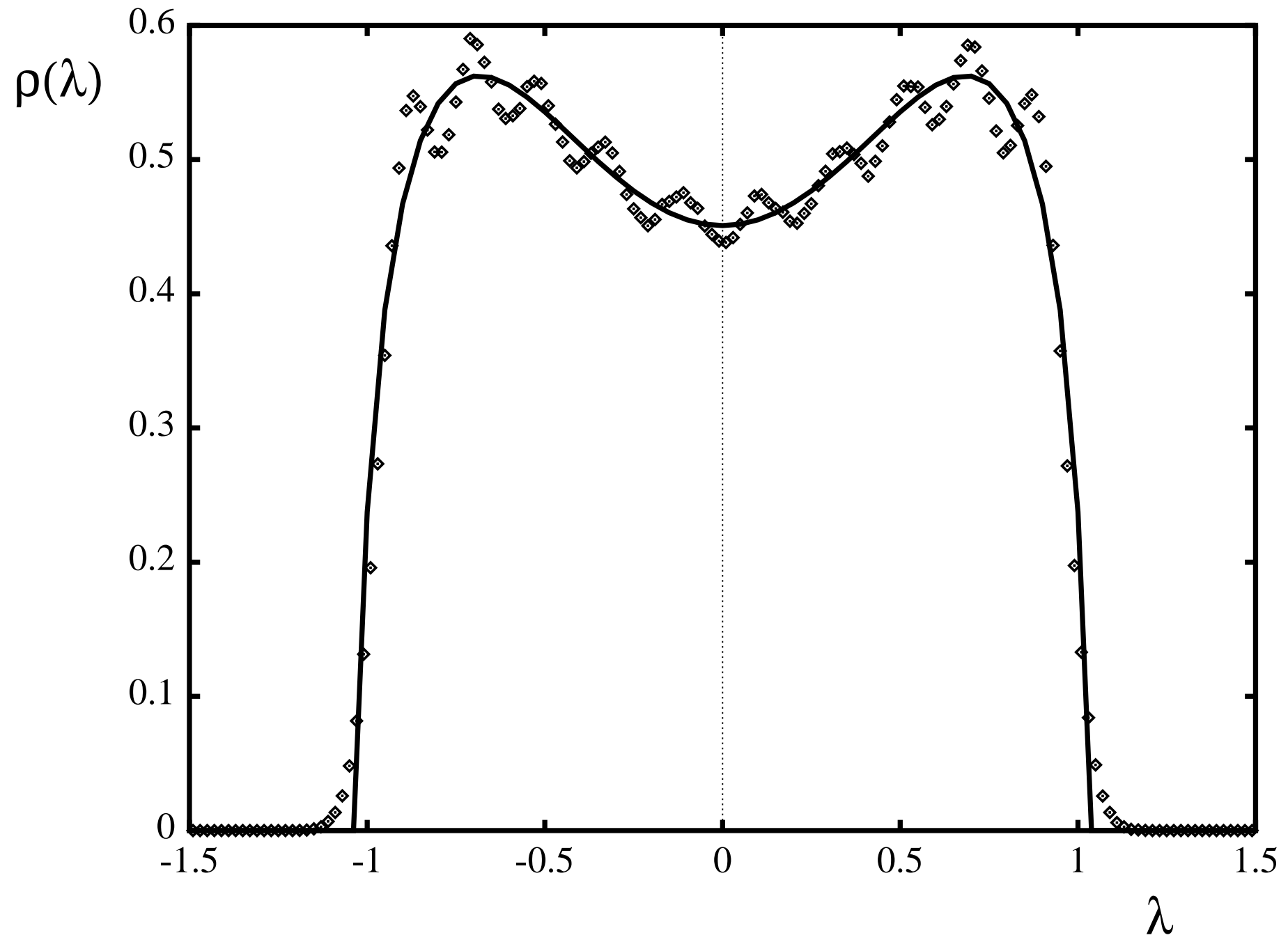

Figure 3 


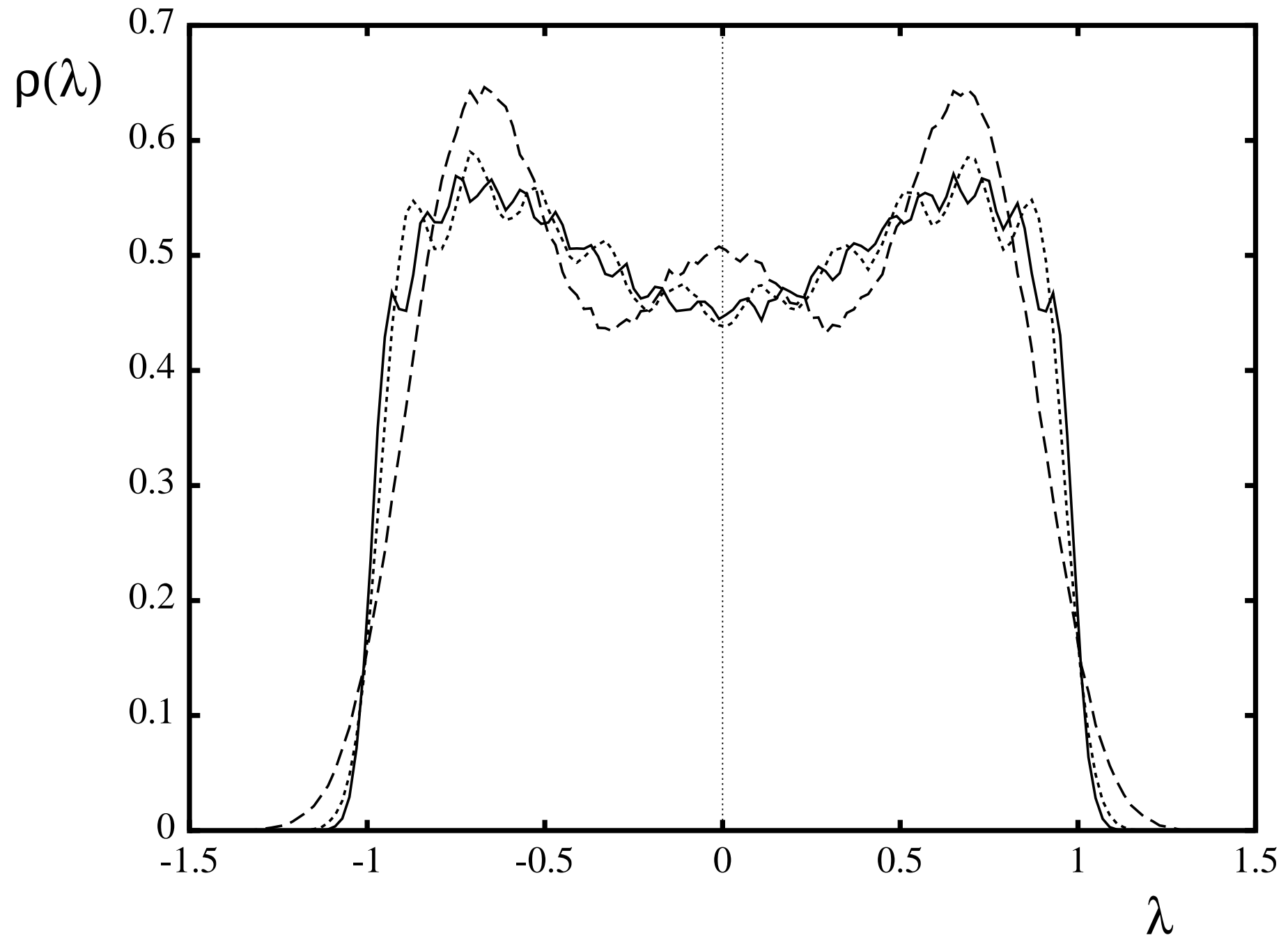

Figure 4 


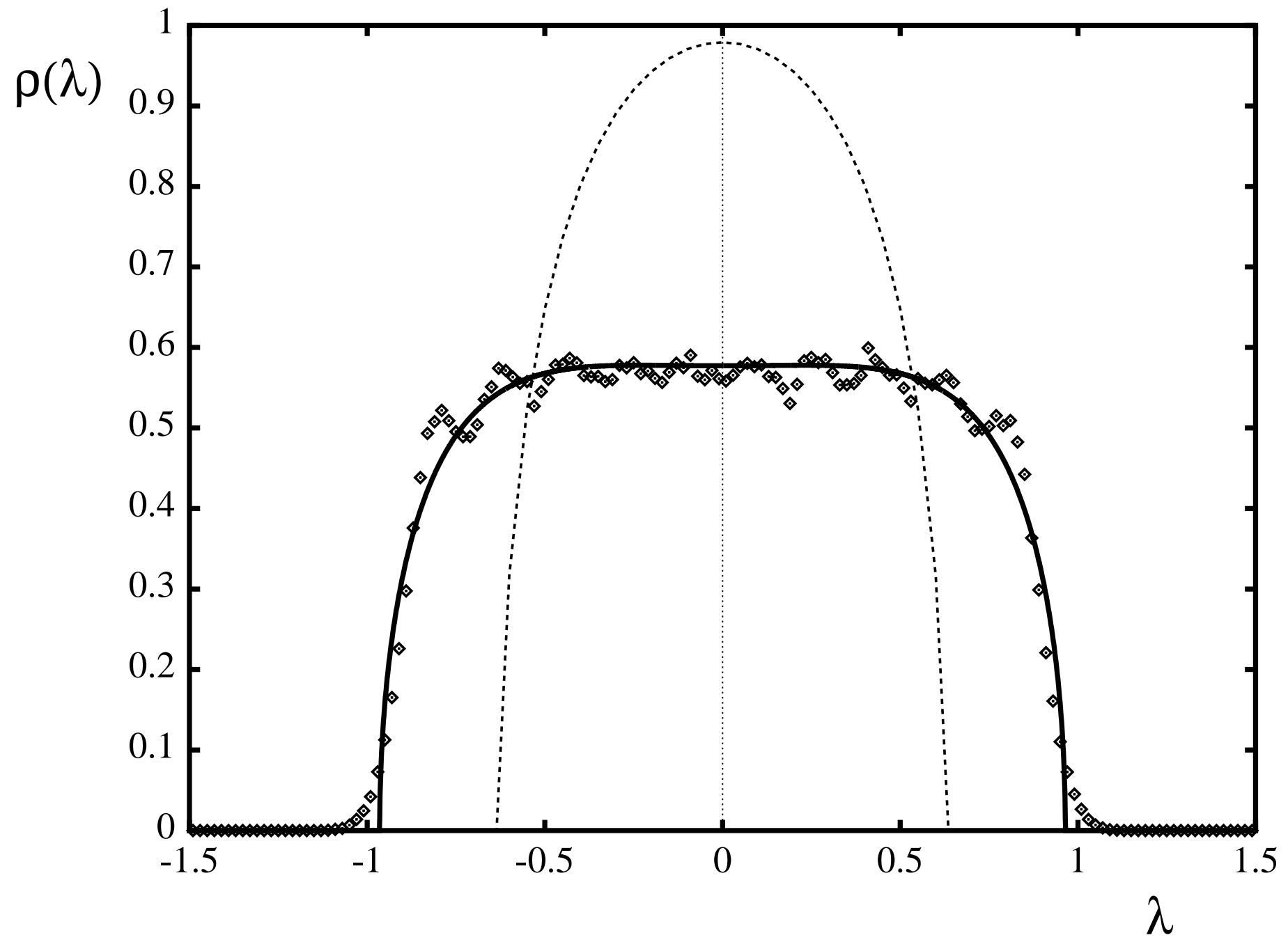

Figure 5 


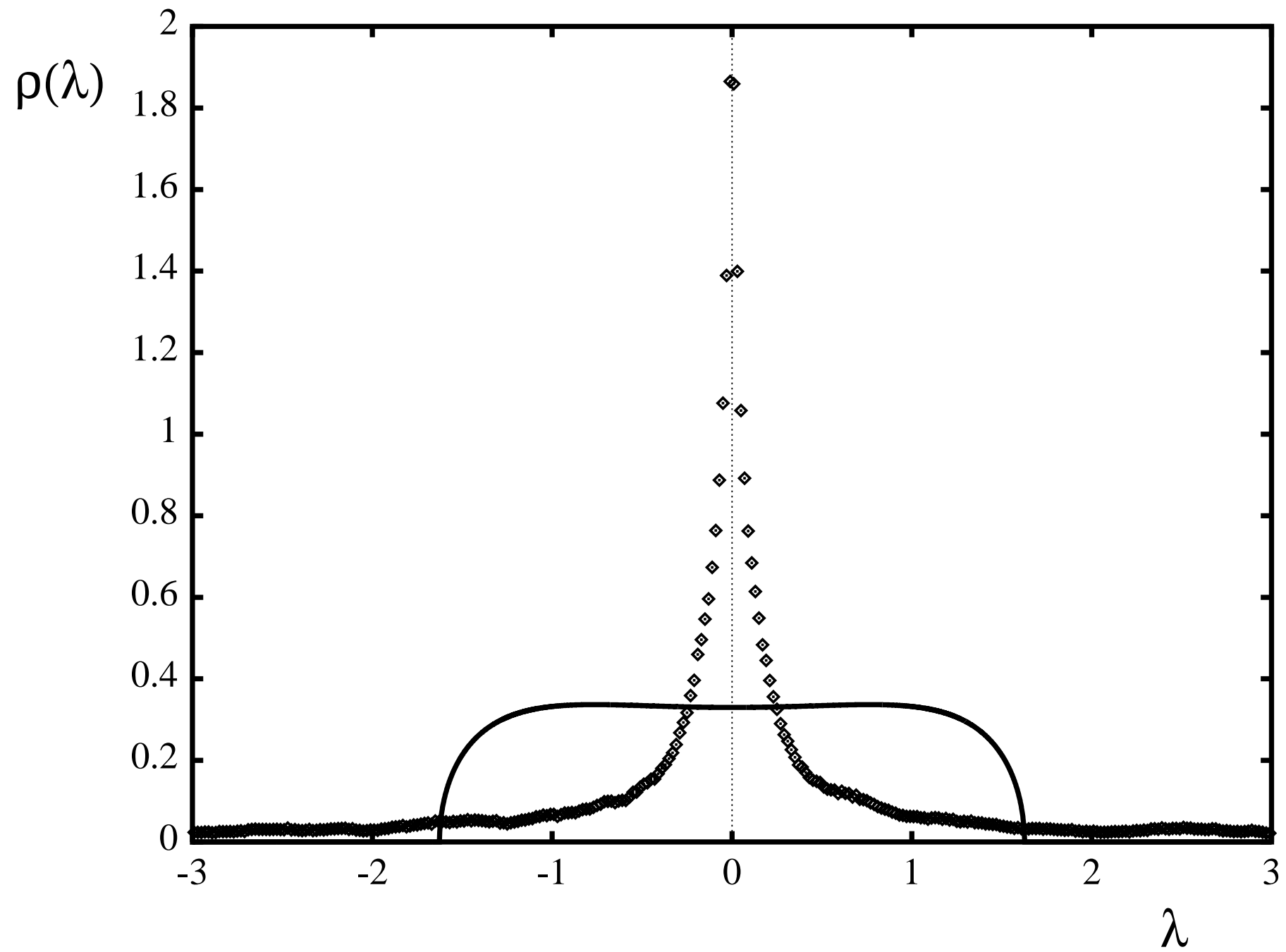

Figure 6 


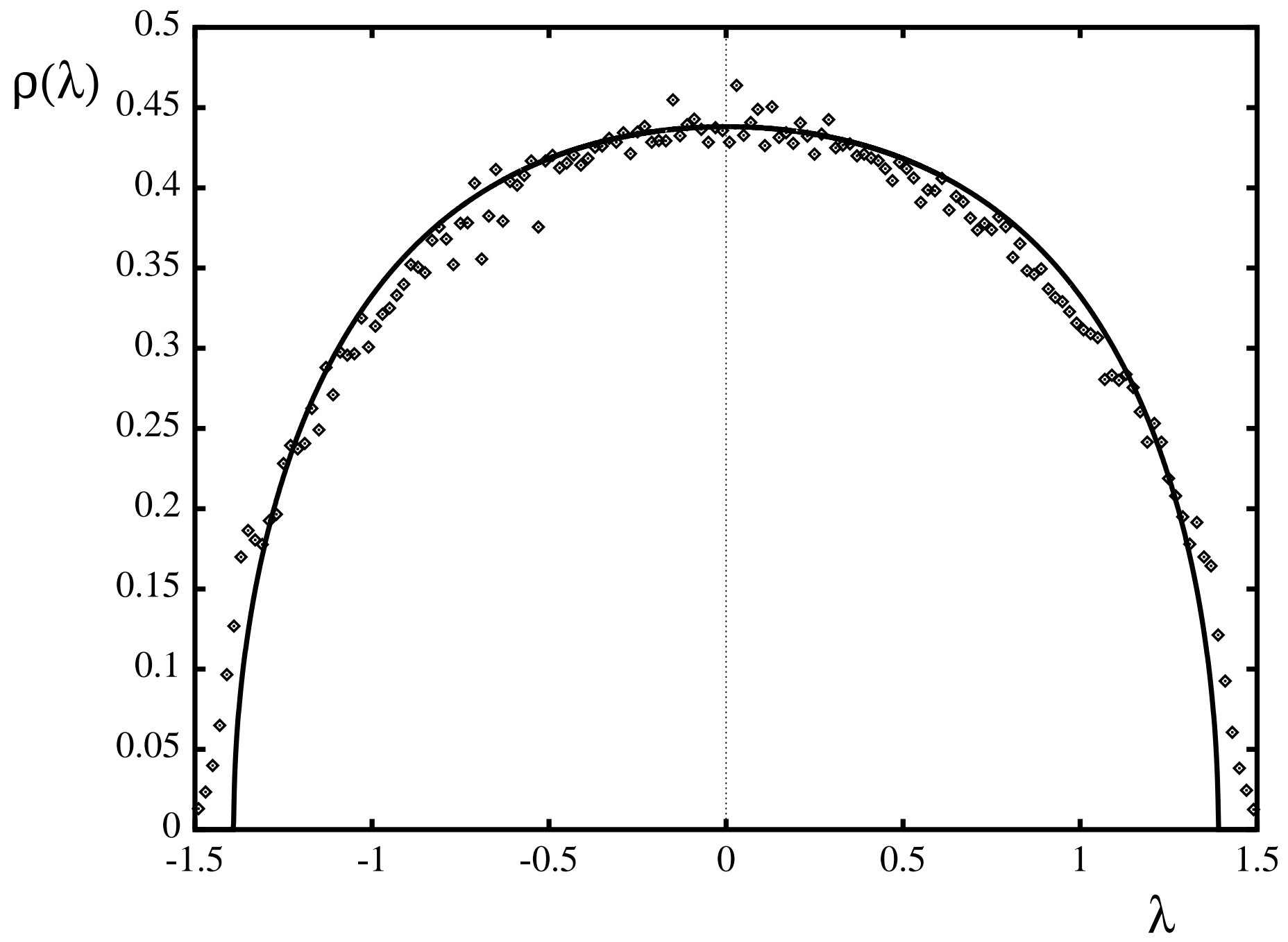

Figure 7 


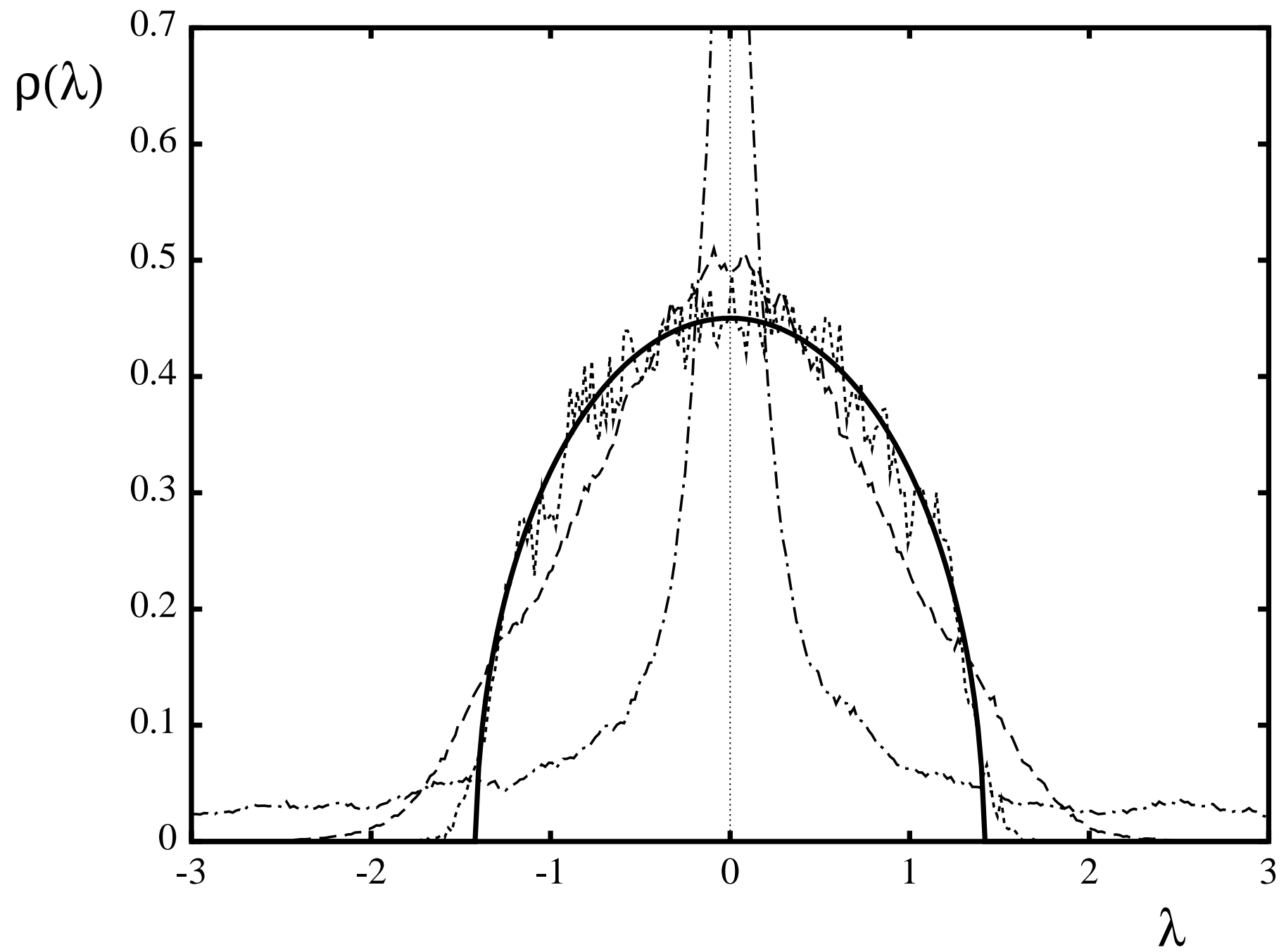

Figure 8 


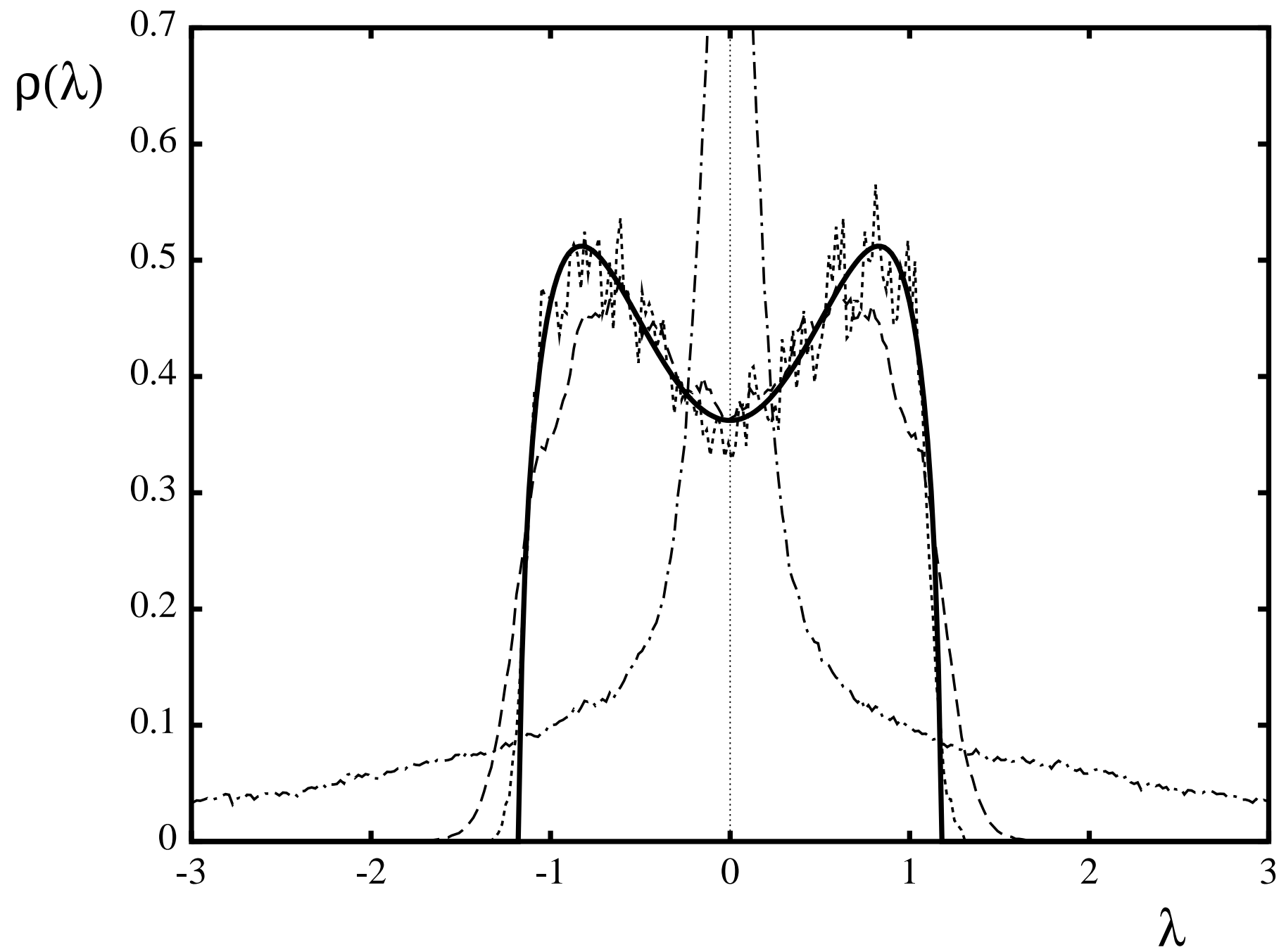

Figure 9 


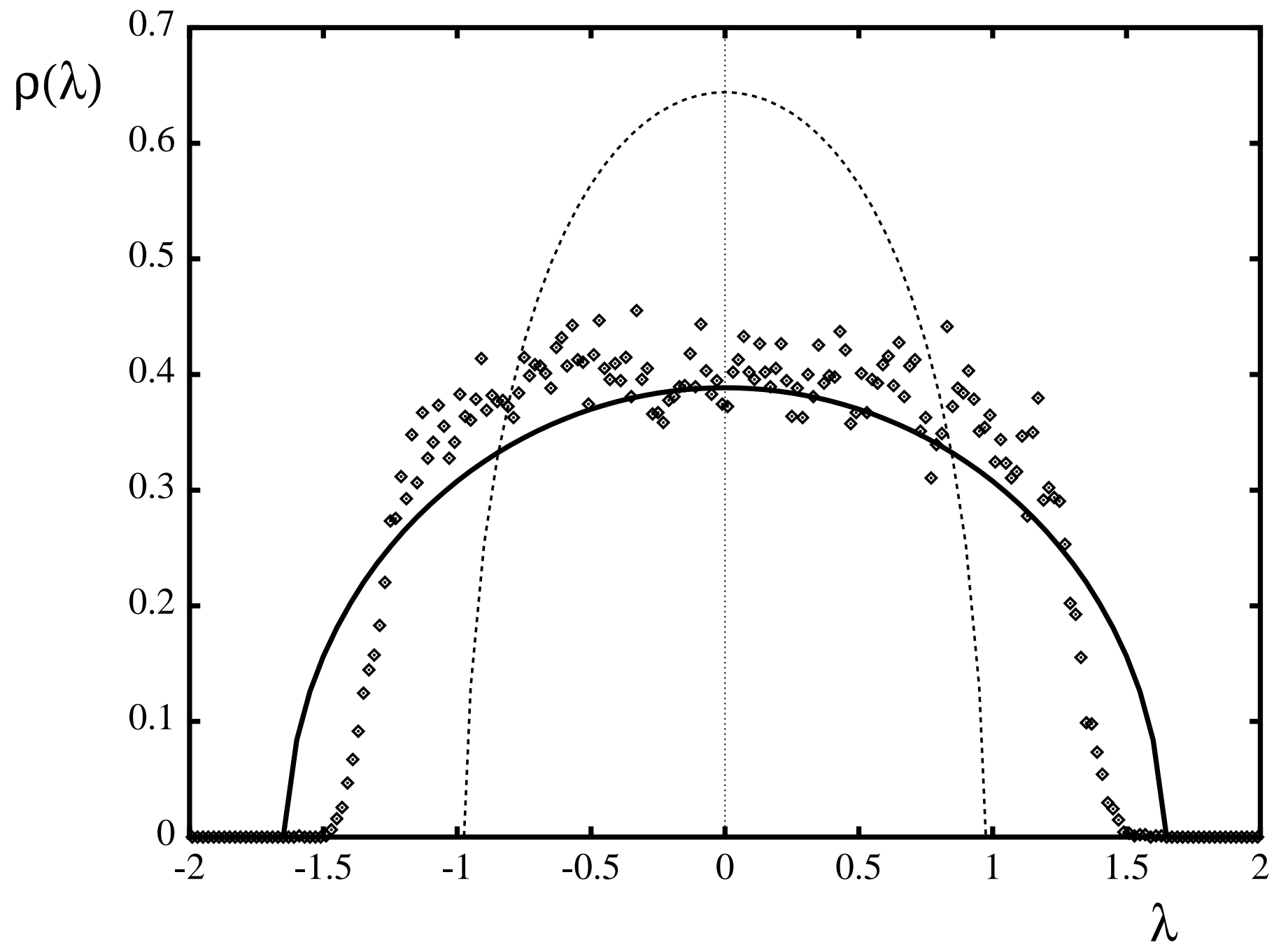

Figure 10 


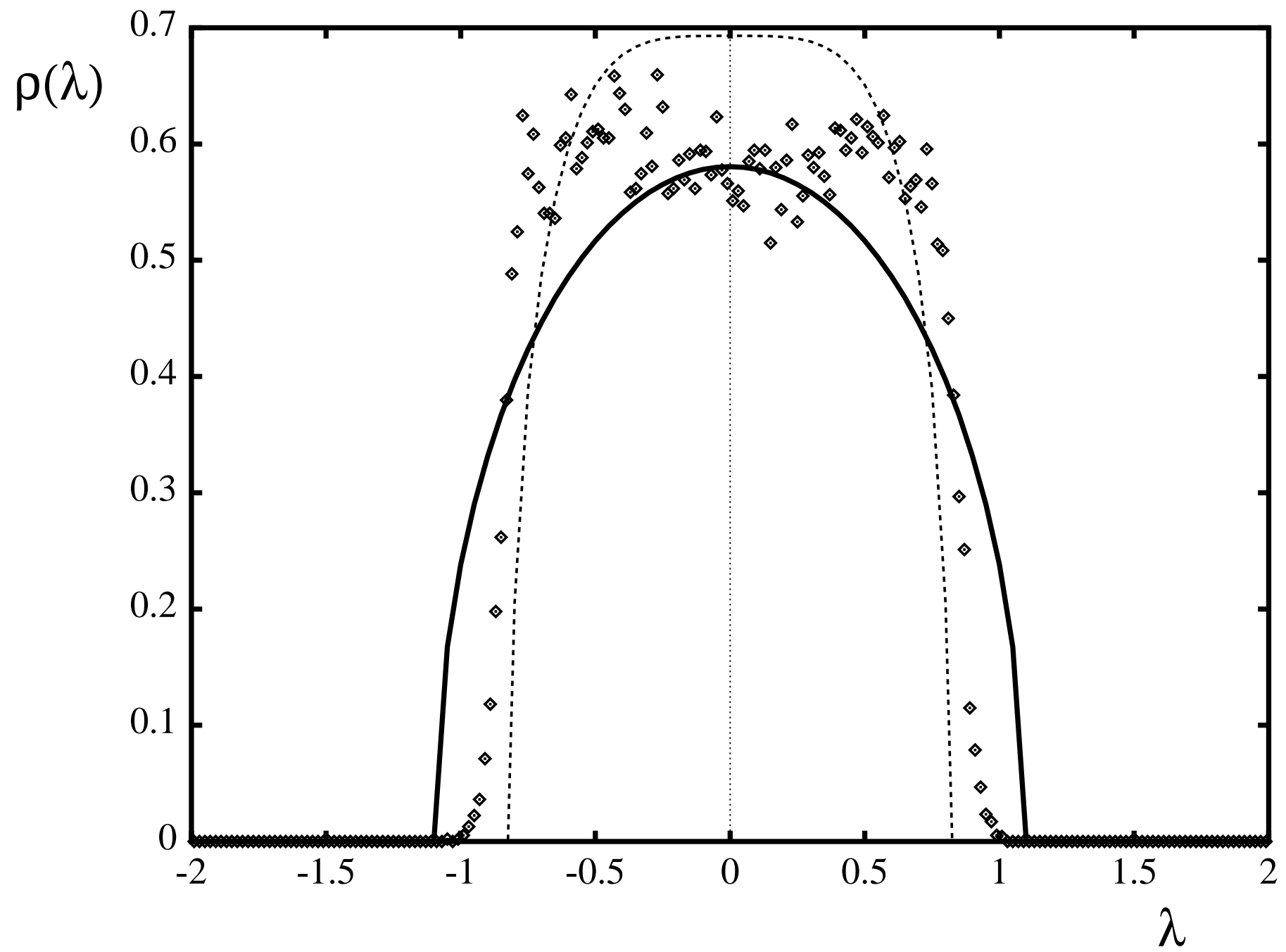

Figure 11 


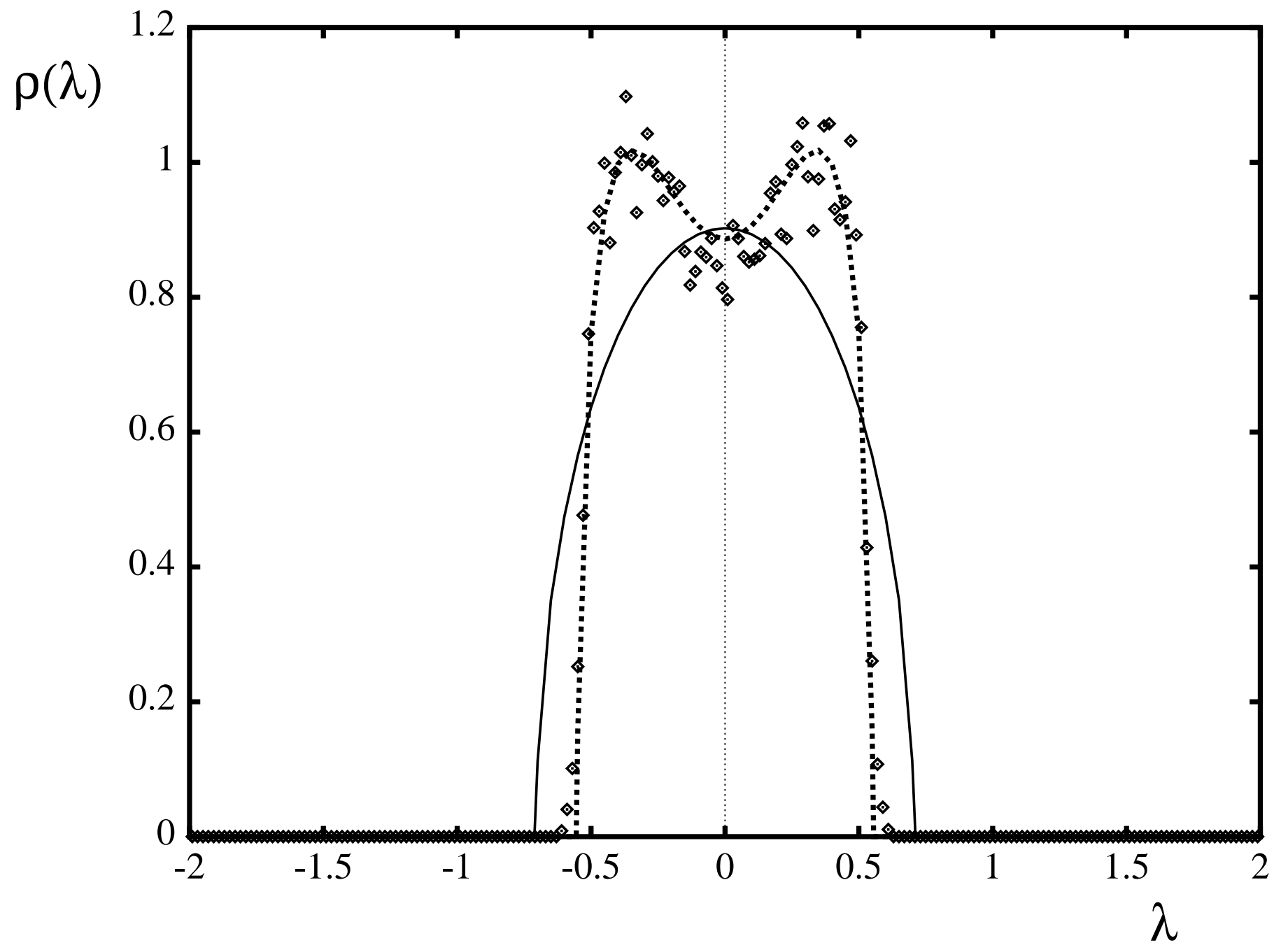

Figure 12 


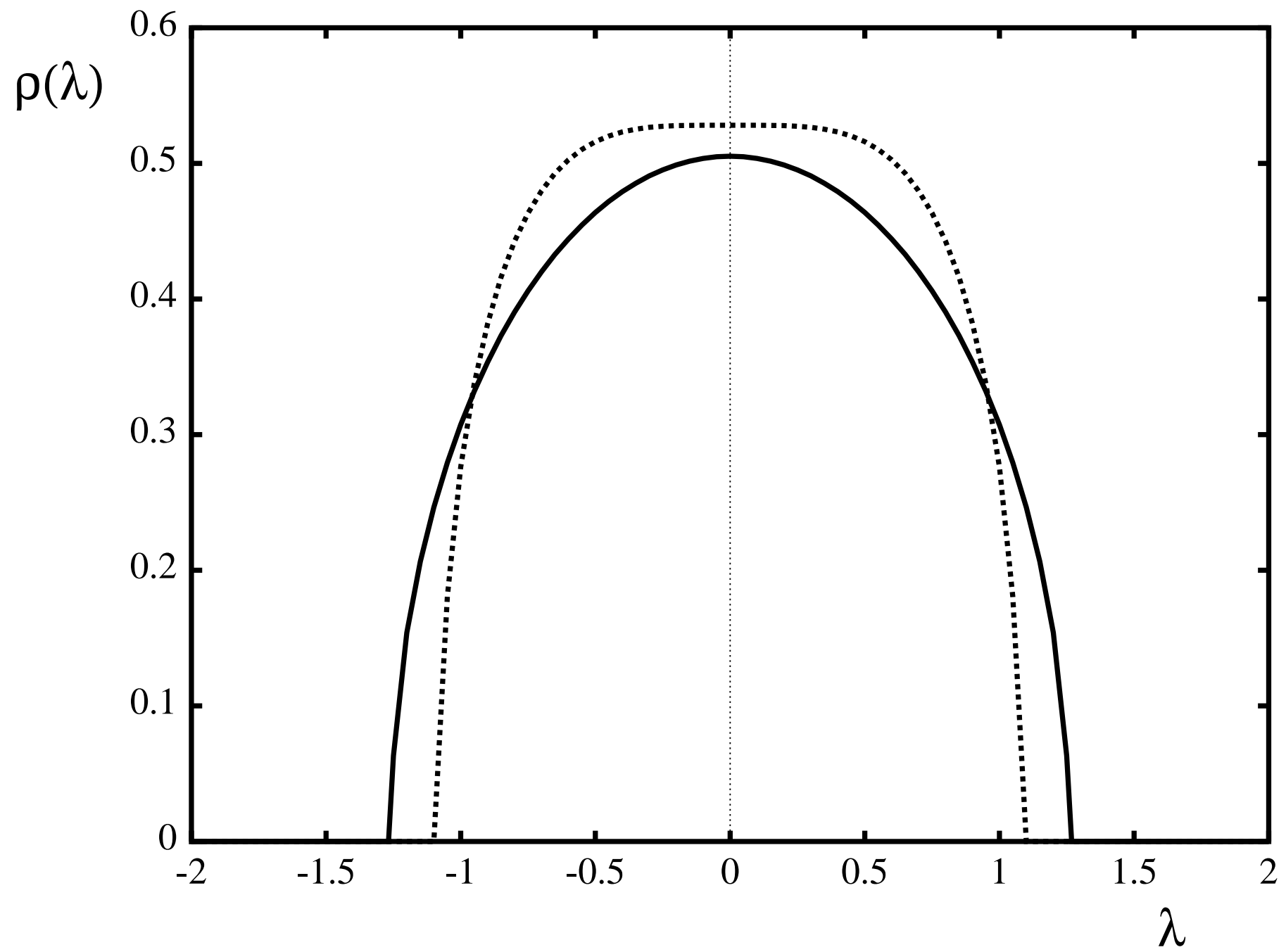

Figure 13 


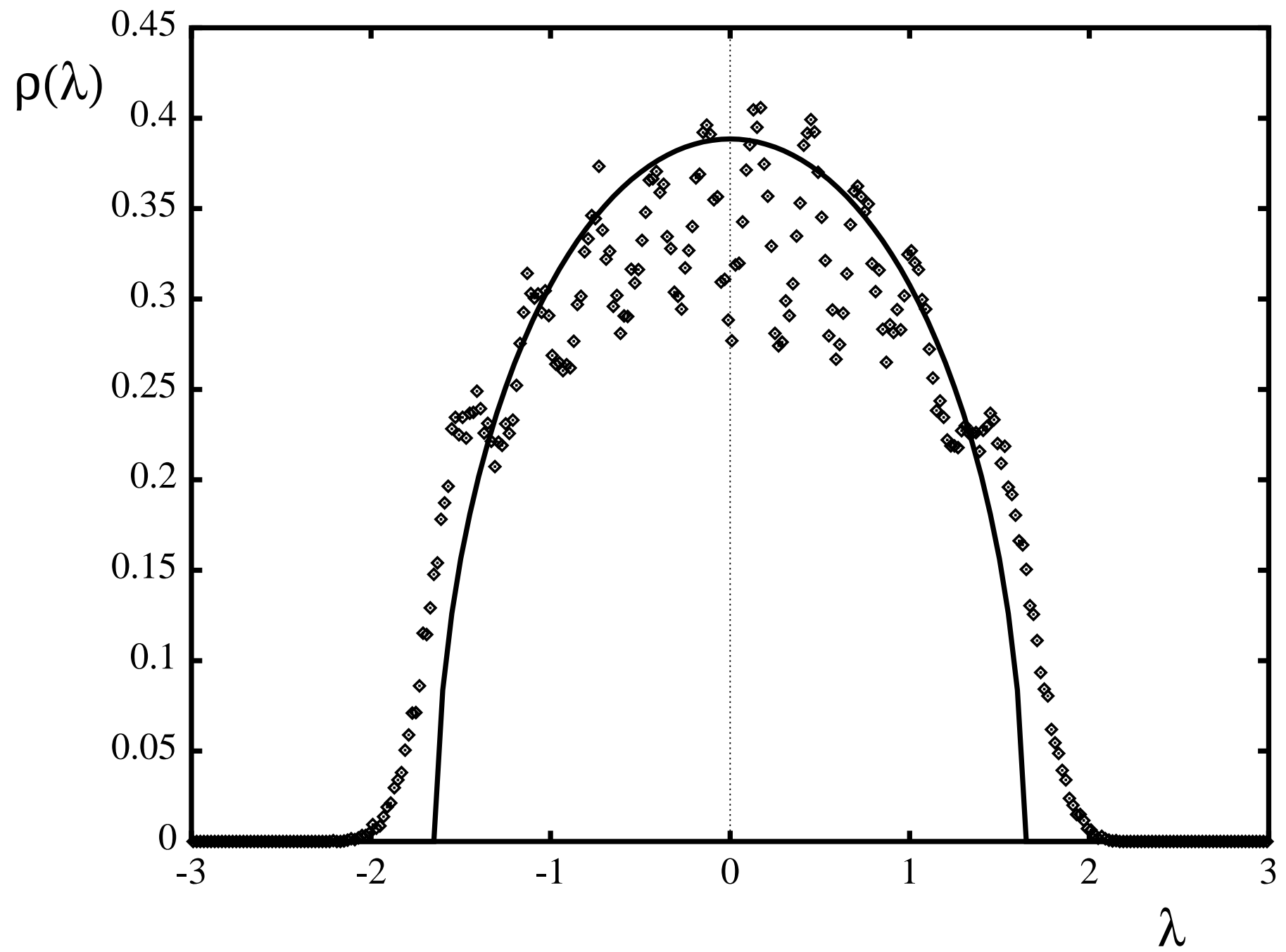

Figure 14 


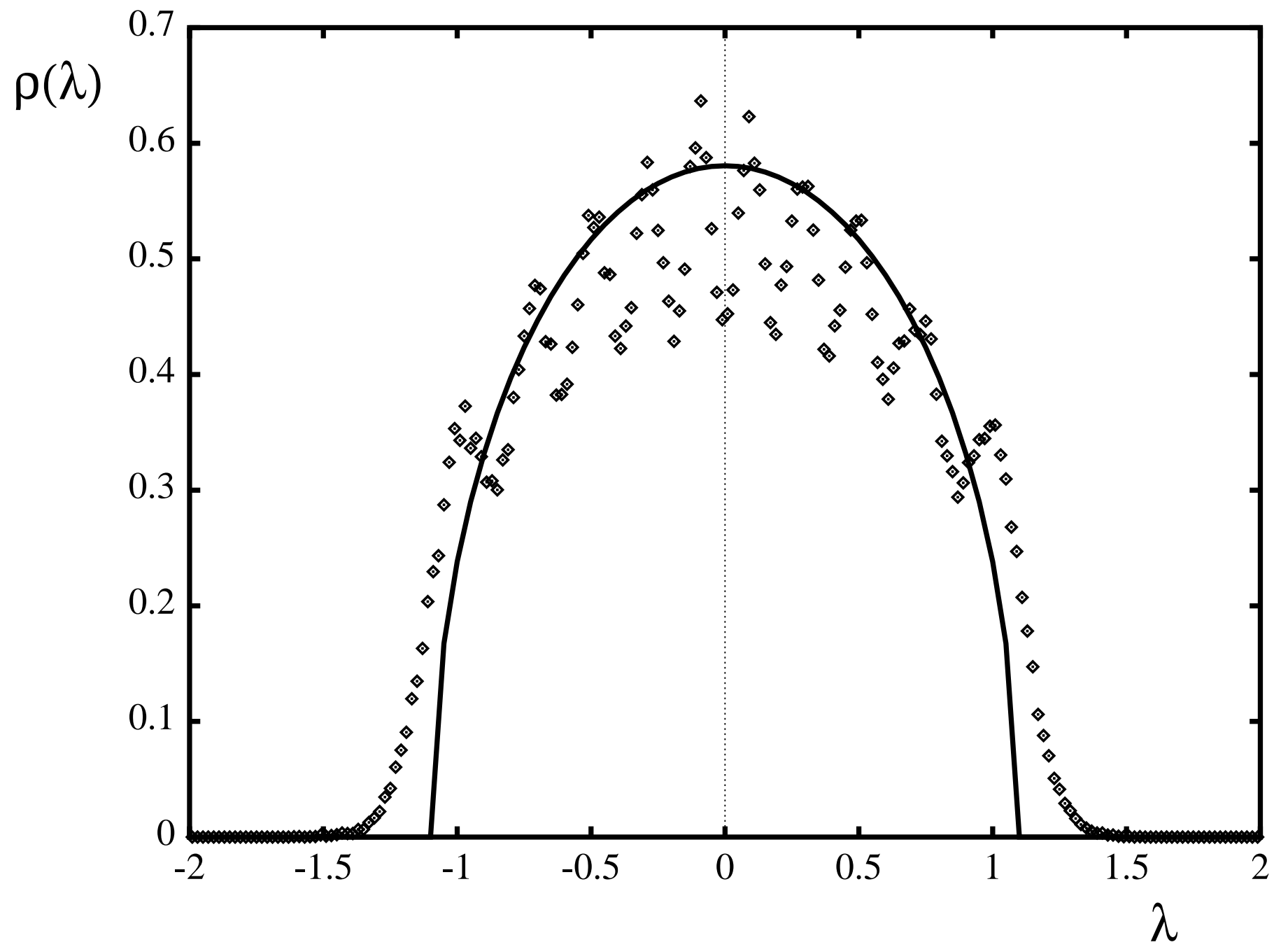

Figure 15 


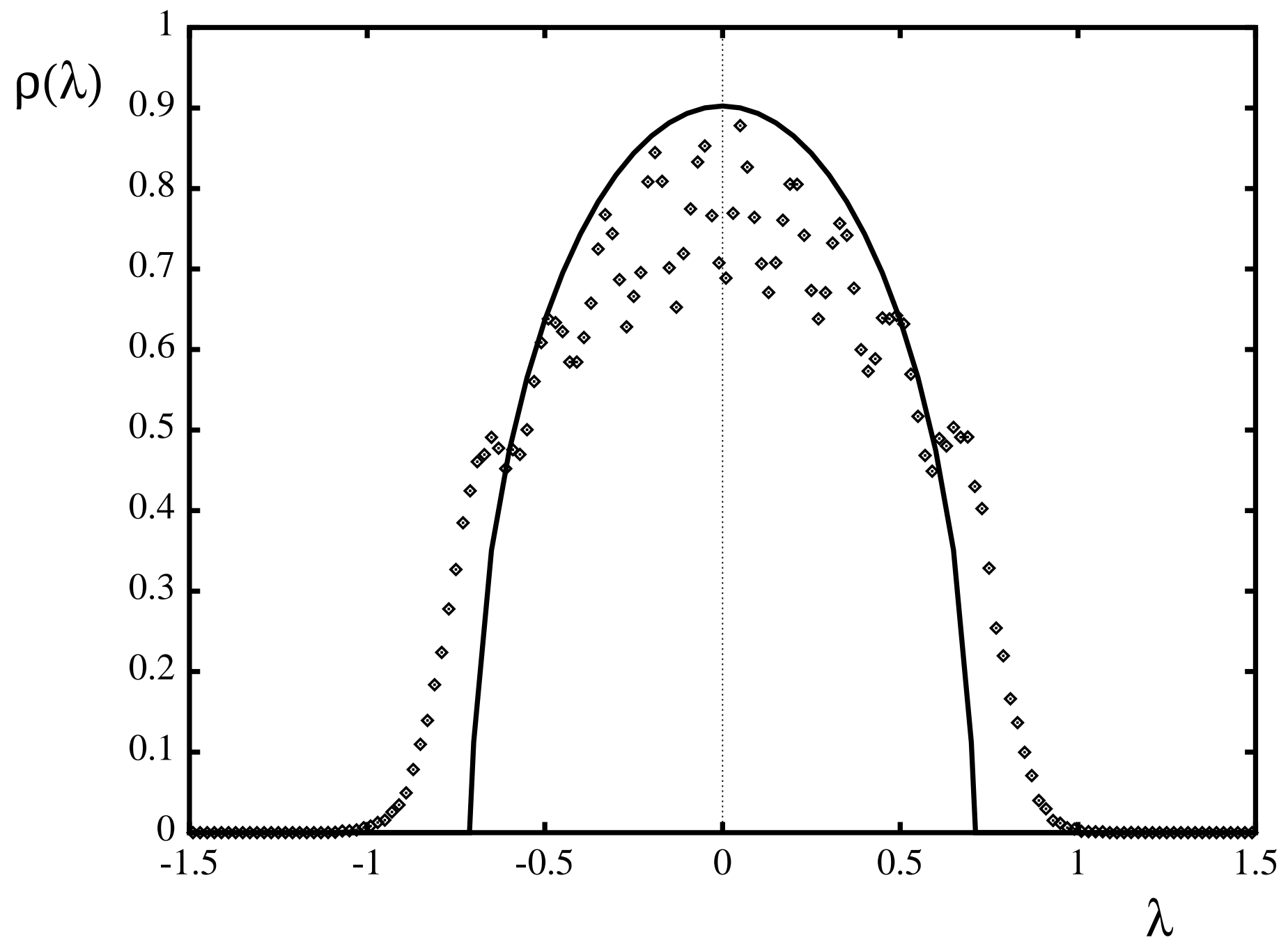

Figure 16 